\title{
The continuous rise of bulges out of galactic disks ${ }^{\star}$
}

\author{
Iris Breda $^{1,2}$ and Polychronis Papaderos ${ }^{1}$
}

\author{
${ }^{1}$ Instituto de Astrofísica e Ciências do Espaço, Universidade do Porto, CAUP, Rua das Estrelas, 4150-762 Porto, Portugal \\ 2 Departamento de Física e Astronomia, Faculdade de Ciências, Universidade do Porto, Rua do Campo Alegre, 4169-007 Porto, \\ Portugal \\ e-mail: iris.breda@astro.up.pt; papaderos@astro.up.pt
}

Received 3 August 2017 / Accepted 30 November 2017

\begin{abstract}
Context. A key subject in extragalactic astronomy concerns the chronology and driving mechanisms of bulge formation in late-type galaxies (LTGs). The standard scenario distinguishes between classical bulges and pseudo-bulges (CBs and PBs, respectively), the first thought to form monolithically prior to disks and the second gradually out of disks. These two bulge formation routes obviously yield antipodal predictions on the bulge age and bulge-to-disk age contrast, both expected to be high (low) in CBs (PBs).

Aims. Our main goal is to explore whether bulges in present-day LTGs segregate into two evolutionary distinct classes, as expected from the standard scenario. Other questions motivating this study center on evolutionary relations between LTG bulges and their hosting disks, and the occurrence of accretion-powered nuclear activity as a function of bulge stellar mass $\mathcal{M}_{\star}$ and stellar surface density $\Sigma_{\star}$.

Methods. In this study, we have combined three techniques - surface photometry, spectral modeling of integral field spectroscopy data and suppression of stellar populations younger than an adjustable age cutoff with the code REMOVEYOUNG $(\mathcal{R} \mathcal{Y})-$ toward a systematic analysis of the physical and evolutionary properties (e.g., $\mathcal{M}_{\star}, \Sigma_{\star}$ and mass-weighted stellar age $\left\langle t_{\star}\right\rangle_{\mathcal{M}}$ and metallicity $\left\langle Z_{\star}\right\rangle_{\mathcal{M}}$, respectively) of a representative sample of 135 nearby ( $\left.\leq 130 \mathrm{Mpc}\right)$ LTGs from the CALIFA survey that cover a range between $10^{8.9} M_{\odot}$ and $10^{11.5} M_{\odot}$ in total stellar mass $\mathcal{M}_{\star, \mathrm{T}}$. In particular, the analysis here revolves around $\left\langle\delta \mu_{9 \mathrm{G}}\right\rangle$, a new distance- and formally extinction-independent measure of the contribution by stellar populations of age $\geq 9$ Gyr to the mean $r$-band surface brightness of the bulge. We argue that $\left\langle\delta \mu_{9 \mathrm{G}}\right\rangle$ offers a handy semi-empirical tracer of the physical and evolutionary properties of LTG bulges and a promising means for their characterization.

Results. The essential insight from this study is that LTG bulges form over 3 dex in $\mathcal{M}_{\star}$ and more than 1 dex in $\Sigma_{\star}$ a tight continuous

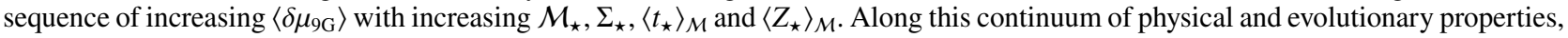
our sample spans a range of $\sim 4 \mathrm{mag}$ in $\left\langle\delta \mu_{9 \mathrm{G}}\right\rangle$ : high- $\left\langle\delta \mu_{9 \mathrm{G}}\right\rangle$ bulges are the oldest, densest and most massive ones $\left(\left\langle t_{\star}\right\rangle_{\mathcal{M}} \sim 11.7 \mathrm{Gyr}\right.$, $\left.\Sigma_{\star}>10^{9} M_{\odot} \mathrm{kpc}^{-2}, \mathcal{M}_{\star} \geq 10^{10} M_{\odot}\right)$, whereas the opposite is the case for low- $\left\langle\delta \mu_{9 \mathrm{G}}\right\rangle$ bulges $\left(\left\langle t_{\star}\right\rangle_{\mathcal{M}} \sim 7 \mathrm{Gyr}\right)$ that generally reside in low-mass LTGs. Furthermore, we find that the bulge-to-disk age and metallicity contrast, as well as the bulge-to-disk mass ratio, show a positive trend with $\mathcal{M}_{\star, \mathrm{T}}$, raising from, respectively, $\sim 0 \mathrm{Gyr}, \sim 0$ dex and 0.25 to $\sim 3 \mathrm{Gyr}, \sim 0.3$ dex and 0.67 across the mass range covered by our sample. Whereas gas excitation in lower-mass $\left(\lesssim 10^{9.7} M_{\odot}\right)$ bulges is invariably dominated by star formation (SF), LINER- and Seyfert-specific emission-line ratios were exclusively documented in high-mass $\left(\gtrsim 10^{10} M_{\odot}\right)$, high- $\Sigma_{\star}\left(\gtrsim 10^{9} M_{\odot} \mathrm{kpc}^{-2}\right)$ bulges. This is in agreement with previous work and consistent with the notion that the Eddington ratio or the black hole-to-bulge mass ratio scale with $\mathcal{M}_{\star}$. The coexistence of Seyfert and SF activity in $\sim 20 \%$ of higher- $\mathcal{M}_{\star}$, high- $\Sigma_{\star}$ bulges being spectroscopically classified as Composites suggests that the onset of AGN-driven feedback does not necessarily lead to an abrupt termination of SF in LTG nuclei.

Conclusions. The continuity both in the properties of LTG bulges themselves and in their age and metallicity contrast to their parent disks suggests that these components evolve alongside in a concurrent process that leads to a continuum of physical and evolutionary characteristics. Our results are consistent with a picture where bulge growth in LTGs is driven by a superposition of quick-early and slow-secular processes, the relative importance of which increases with $\mathcal{M}_{\star, \mathrm{T}}$. These processes, which presumably combine in situ SF in the bulge and inward migration of material from the disk, are expected to lead to a non-homologous radial growth of $\Sigma_{\star}$ and a trend for an increasing Sérsic index with increasing galaxy mass.
\end{abstract}

Key words. galaxies: spiral - galaxies: bulges - galaxies: evolution

\section{Introduction}

The driving mechanisms and chronology of the buildup of bulges in late-type galaxies (LTGs) is an issue of key relevance to our understanding of galaxy evolution. According to our current knowledge on bulge demographics in the local universe, a large fraction of LTGs host pseudo-bulges (PBs; e.g., Gadotti 2009;

* Table C.1 is only available at the CDS via anonymous ftp to cdsarc.u-strasbg.fr $(130.79 .128 .5)$ or via http://cdsarc.u-strasbg.fr/viz-bin/qcat?J/A+A/vol/page
Fisher \& Drory 2011; Fernández Lorenzo et al. 2014) that substantially differ from classical bulges (CBs) in their spectrophotometric and kinematical characteristics. The latter resemble in many respects "old and dead" elliptical galaxies, lacking ongoing star-formation (SF), exhibit a spheroidal shape with inwardly steeply increasing surface brightness profiles (SBPs) being well approximated by the Sérsic (1963) fitting law with a high $(\gtrsim 3)$ exponent $\eta$, show stellar kinematics dominated by velocity dispersion $\left(\sigma_{\star}\right)$ and obey the Kormendy (1977) scaling relations for normal elliptical galaxies (Fisher \& Drory 2010). It is observationally established that $\mathrm{CBs}$ contain a super-massive black 
hole (SMBH) with a mass $M_{\bullet}$ tightly correlating with their stellar mass $\mathcal{M}_{\star, \mathrm{B}}, \sigma_{*}$ and optical luminosity (Ho 2008; Kormendy \& Ho 2013; see also Ferrarese \& Merritt 2000). Traditionally, bulges were thought to invariably form early-on via violent quasi-monolithic gas collapse (Larson 1974) or mergers (Bender et al. 1992; Aguerri et al. 2001; Keselman \& Nusser 2012) associated with vigorous nuclear starbursts (Okamoto 2012), with the disk gradually building up around them. Whereas this insideout galaxy formation scenario appears consistent with important integral characteristics of CBs (e.g., their red colors), it does not offer a plausible explanation for the presence of PBs in present-day LTGs. These generally show ongoing SF, a significant degree of rotational support (Kormendy \& Kennicutt 2004, for a review) and flatter/ellipsoidal shapes with nearly exponential SBPs $(\eta \lesssim 2$; e.g., Drory \& Fisher 2007; Fisher \& Drory 2010). Even though there is observational evidence that PBs also contain a SMBH (Kormendy et al. 2011; Kormendy \& Ho 2013), in some cases revealing itself as an active galactic nucleus (AGN; e.g., Kotilainen et al. 2016; see Kormendy \& Ho 2013 for a review), these do not follow the $M_{\bullet}-\sigma_{*}$ correlation for CBs, which appears to be consistent with a different formation route. Indeed, the prevailing concept on PB formation is that these entities emerge gradually out of galactic disks through gentle gas inflow spawning quasi-continuous SF and the emergence of a central bulge-like luminosity excess at their centers (e.g., Courteau et al. 1996; Carollo et al. 2001; Kormendy \& Kennicutt 2004). Besides bar-driven gas inflow (e.g., Springel \& Hernquist 2005), various other mechanisms, such as inward stellar migration, minor mergers with low-mass satellites, or a purely dynamical re-arrangement of the disk (Scannapieco et al. 2010; Guedes et al. 2013; Bird et al. 2012; Roškar et al. 2012; Grand et al. 2014; Halle et al. 2015) have been proposed as further contributors to PB growth along the Gyr-long secular evolution of LTGs.

These two scenarios, the first one envisaging bulge formation prior to disks and the second one out of disks obviously yield antipodal predictions on the bulge age and bulge-to-disk color contrast, both expected to be high (low) in CBs (PBs). Naively, one might therefore expect bulges in present-day LTGs to describe a bimodal age distribution, with each of the two classes showing a high degree of homogeneity in its spectrophotometric, chemodynamical and evolutionary properties, echoing two distinct formation routes. However, the available observational data contrast the picture of an age bimodality in LTG bulges, suggesting instead a substantial spread, if not smooth transition, in their properties across their mass and luminosity range. For example, Wyse et al. (1997), reviewing the subject two decades ago, conclude that bulges show a considerable heterogeneity, with merely higher luminosity ones (CBs) having a closer affinity with Ellipticals and lower luminosity bulges (PBs) with disks.

From the perspective of the monolithic bulge formation scenario, there is evidently no other option than to interpret PBs as SF-rejuvenated CBs, and indeed, this proposal has been put forward in several studies (e.g., Thomas \& Davies 2006; Johnston et al. 2012, 2014; Morelli et al. 2012, 2016). For example, Thomas \& Davies (2006) drew this conclusion by reproducing a subset of Lick indices $\left(\mathrm{H} \beta, \mathrm{H} \gamma \mathrm{A}, \mathrm{H} \delta_{\mathrm{A}},[\mathrm{MgFe}]^{\prime}\right.$, and $\langle\mathrm{Fe}\rangle$ ) for local bulges with a two-component evolutionary synthesis (ESS) model that involves a 15 Gyr old stellar component, which 1-2 Gyr ago underwent a SF episode producing up to $10-30 \%$ of its total $\mathcal{M}_{\star}$. On the basis, of these models and observed correlations between central $\sigma_{\star}\left(\right.$ aka $\left.\mathcal{M}_{\star, T}\right)$ and the luminosity-weighted age, metallicity and $\alpha / \mathrm{Fe}$ ratio, these authors argue that the smallest bulges are the youngest and have experienced a late iron enrichment by type Ia SNe.

Intermediate between the "quick" (monolithic) and "slow" (secular) bulge formation scenarios above is a set of models envisaging the dominant phase of bulge formation to occur in a prolonged episode of $\sim 0.3-0.8 \mathrm{Gyr}$ through inward migration and coalescence of massive $\left(\gtrsim 10^{8-9} M_{\odot}\right)$ SF clumps forming continuously in the disk out of gas instabilities (Noguchi 1999; Bournaud et al. 2007; Carollo et al. 2007; Elmegreen et al. 2008; Mandelker et al. 2014, 2017). Observational support for this picture comes from the clumpiness of high- $z$ proto-disks and the estimated $\mathcal{M}_{\star}$ of their SF clumps (Förster Schreiber et al. 2011; Wuyts et al. 2012). Additionally, inflowing inter-clump gas (e.g., Hopkins et al. 2012; Zolotov et al. 2015) could sustain perpetual rejuvenation of the bulge with in situ SF, which acts together with stellar migration, minor mergers with dwarf satellites and other dynamical effects (e.g., Bird et al. 2012; Roškar et al. 2012; Guedes et al. 2013; Grand et al. 2014; Halle et al. 2015) toward bulge buildup in the ensuing Gyr of galactic evolution. Depending on the timescales and the contribution of these different processes, it might be expected this set of prolonged bulge formation scenarios to yield a range of stellar ages in present-day LTG bulges.

From the observational point of view, the notion of a correlated evolution of disk and bulge has been put forward is several studies, mostly on the basis of photometric investigations of local LTGs (e.g., Gadotti \& dos Anjos 2001; Gadotti 2009). For example, early work by Peletier \& Balcells (1996) finds that "color variations from galaxy to galaxy are much larger than color differences between disk and bulge in each galaxy", following that "the underlying old population of disks and bulges is much more similar" than previously thought. Also, other works find the color contrast between bulge and disk to be relatively small (e.g., $0.3 \mathrm{~g}-i \mathrm{mag}$ for isolated galaxies studied in Fernández Lorenzo et al. 2014) and interpret this as evidence for a correlated evolution of these components (see also, e.g., Courteau et al. 1996; Gadotti \& dos Anjos 2001). Similarly, a photometric study by Carollo et al. (2007) finds that the colors of bulges are correlated with those of the disks in which they are embedded, suggesting that an early phase in bulge formation must have been supplemented through "continuing" rejuvenation, and in some cases even that the bulk of the stellar mass in bulges has assembled more recently than the disk. These authors also point out that the scaling relations between bulge stellar age and bulge/galaxy mass hint at similar formation processes for all components, suggesting that bulges across their entire range in mass and age result from the internal evolution of the parent disks. They also show that dynamical friction of massive clumps in gas-rich disks offers an explanation for the formation of late-type bulges, especially for those that are older than their surrounding disks, consistently with the picture above. Coming to our own Milky Way (MW), Ness et al. (2014) demonstrate by means of N-body and smooth particle hydrodynamics simulations that the presence of young stars predominantly near the plane is expected for a bulge that has formed from the disk via dynamical instabilities, whereas it cannot be accounted for by monolithic collapse.

The picture of a concomitant evolution of bulge and disk has also received support from HST imaging studies of MW analogs at higher $z$ : van Dokkum et al. (2013), by applying the abundance matching technique to MW-progenitor candidates out to $z=$ 2.5 point out that there is an absence of high-density "naked bulges" at $z \sim 2$, around which disks subsequently assemble (see also Patel et al. 2013). These authors also find that MW-like LTGs have built $\sim 90 \%$ of their present $\mathcal{M}_{\star}$ since $z=2.5$ with 
most of the SF occurring before $z=1$. They verify that for $1<z<2.5$ the mass in the central $2 \mathrm{kpc}$ of MW progenitors increases by a factor of $3.2_{-0.7}^{+0.8}$, which rules out models in which bulges were fully assembled first and disks gradually formed around them. In this context, the question of how the bulge and the disk grew relative to each other since this early cosmic epoch is of considerable interest. Margalef-Bentabol et al. (2016) find from HST photometry of 1495 massive galaxies in the CANDELS field that two-component (bulge and disk) systems in the redshift interval $1.5<z<3$ roughly maintain their bulge size whereas their disks grow by a factor of approximately 3 . A subsequent study of such two-component galaxies in Margalef-Bentabol et al. (2018) finds that the $\mathcal{M}_{\star}$ enclosed within the bulge and disk remains nearly constant over the latter redshift interval. This, and the fact that the disk shows a higher star formation rate (SFR) than the bulge led these authors to conclude that about one half of the stellar mass formed in the disk must have migrated into the bulge, promoting its gradual growth. We note though that evolution of the bulge-to-disk mass ratio across $z$ remains a subject of investigation. For example, Tacchella et al. (2018) report that galaxies with $\mathcal{M}_{\star} \leq 10^{11} M_{\odot}$ have since $z \sim 2$ on average doubled their stellar mass throughout their radial extent.

Whereas most lines of evidence point to an interwoven evolution of bulge and disk, our understanding on how this process has shaped the heterogeneity of present-day bulges is far from complete. In fact, neither observations nor theory yield as yet clear-cut discriminators between CBs and PBs, or even unambiguous evidence for an evolutionary dichotomy between both. From the photometric point of view, it is common practice to classify bulges by their Sérsic index $\eta$, which is assumed to be $>(\leq) \sim 2$ for CBs (PBs). However, this cutoff has no clear physical foundation (e.g., Gadotti 2012) and essentially rests on subjective considerations. For instance, Fisher \& Drory (2010, cf. their Fig. 7) find that across the bulge sequence there is a substantial overlap between CBs and PBs, depending on whether they are selected according to mid-infrared colors or $\eta$. Likewise, some photometric classification clues for PBs merely rely on the absence of correlations that are known to apply to CBs: Fisher \& Drory (2010) find that the $\eta$ of PBs is uncorrelated with other bulge structural properties, unlike CBs. This is in agreement with the analysis of Gadotti (2009) who concludes that CBs follow a correlation between $\eta$ and bulge-to-total (B/T) ratio, whereas PBs do not, and they actually occupy on the fundamental plane the same locus as disks. In this regard, a proposal made by Gadotti (2009) is that a better separation between CBs and PBs is possible on the basis of deviations from the Kormendy (1977) relation rather than on $\eta$. On the other hand, this study has shown that $\mathrm{CBs}$ are offset from Ellipticals in the mass-size relation, suggesting that the former are not simply Ellipticals surrounded by disks. The search for discriminators between CBs and PBs continues attracting considerable interest, with new schemes proposed, such as, for example, that by Neumann et al. (2017), which uses a combination of $\eta$, light concentration index $C_{20,50}$, the Kormendy (1977) relation and the inner slope of the radial $\sigma_{\star}$ profile.

As for spectral modeling studies of single-fiber SDSS data, they find a trend for increasing bulge age and metallicity with increasing $\mathcal{M}_{\star, T}$, with CBs (PBs) generally populating the high (low) range of a broad sequence in mass (e.g., Zhao 2012; Ribeiro et al. 2016). These trends are essentially echoing relations obtained or corroborated over the past decade from spectral fitting of SDSS data for large extragalactic probes, as for example, a positive correlation between $\mathcal{M}_{\star, T}$, gas-phase and stellar metallicity $\left(Z_{\mathrm{g}}\right.$ and $Z_{\star}$, respectively) and light- and mass-weighted stellar age $\left(\left\langle t_{\star}\right\rangle_{\mathcal{L}}\right.$ and $\left\langle t_{\star}\right\rangle_{\mathcal{M}}$, respectively; e.g., Kauffmann et al. 2013; Tremonti et al. 2004). They are also consistent with the galaxy "downsizing" picture, reflected in, for example, an anti-correlation between $\mathcal{M}_{\star, T}$ with specific star formation rate (sSFR) and $\left\langle t_{\star}\right\rangle_{\mathcal{L}}$ for local galaxies (e.g., Brinchmann et al. 2004; Heavens et al. 2004; Gallazzi et al. 2005; Noeske et al. 2007; Asari et al. 2007), which implies that massive galaxies have experienced the dominant phase of their assembly early on, whereas lower-mass systems build up their stellar mass over longer timescales. For instance, Gallazzi et al. (2005) find a sequence of increasing $\mathcal{M}_{\star, T}$ and $\left\langle t_{\star}\right\rangle_{\mathcal{L}}$ with increasing light-weighted $Z_{\star}\left(\left\langle Z_{\star}\right\rangle_{\mathcal{L}}\right)$ and $4000 \AA$ break strength, indicating that low-mass galaxies are typically younger and less metal-enriched, contrary to massive ones, with a transition between these two regimes occurring at $3 \times 10^{9}<\mathcal{M}_{\star, T}$ $\left(M_{\odot}\right)<3 \times 10^{10}$. It is unclear though whether the absence of sensitive discriminators between CBs and PBs, despite intense exploration of spectroscopic SDSS data over the past years, is due to a genuine continuity in the physical and evolutionary properties (e.g., $\mathcal{M}_{\star}$ and $\left\langle t_{\star}\right\rangle_{\mathcal{M}}$, respectively) of LTG bulges, or party because of aperture effects (cf. discussion in, e.g., Gomes et al. 2016b, and references therein). The latter are unavoidable, given that the $3^{\prime \prime}$ fiber of SDSS captures only a small portion of the bulge for nearby LTGs, whereas it also includes the surrounding disk for more distant ones. In the first case, evolutionary characteristics inferred from spectral modeling are representative for the bulge only as long as age and metallicity gradients therein are weak, whereas in the second case they could be systematically biased through the star-forming disk. For the typical bulge radius of a massive LTG in our sample ( $\sim 3 \mathrm{kpc}$; cf. Table C.1) this bias is expected for $z \geq 0.34$ (standard cosmology assumed).

Wide-field integral field spectroscopy (IFS) can in principle overcome these aperture biases, since it allows for spectroscopic analysis of the total bulge emission within a photometrically defined radius obtained from SBP decomposition into bulge and disk. Although recent IFS studies have explored various physical relations between, for example, $\Sigma_{\star},\left\langle t_{\star}\right\rangle_{\mathcal{L}},\left\langle Z_{\star}\right\rangle_{\mathcal{L}}$ and SFR for LTGs as a function of galactocentric radius (e.g., GonzálezDelgado et al. 2014, 2016; Cano-Díaz et al. 2016; Zibetti et al. 2017), they generally did not incorporate a structural analysis that would have permitted extraction and spectral modeling of the total bulge emission within a uniformly defined radius. An exception to this is the work by Sánchez-Blázquez et al. (2014) where the bulge extent was estimated from image decomposition as the radius where the intensity of the bulge equals that of the disk, to which that study was mainly devoted. The normalization of radial profiles for various quantities inferred from these IFS studies to the galaxy effective radius is another possible issue, since this approach bears the risk of comparing determinations within the bulge of higher-B/T LTGs with those in the disk of lower-B/T LTGs (cf. Sect. 2.2.2).

The goal of this study is to explore on the basis of a combined spectral modeling and surface photometry analysis of a representative sample of local LTGs the connection between physical and evolutionary properties of bulges (e.g., $\mathcal{M}_{\star}, \Sigma_{\star}$ and $\left\langle t_{\star}\right\rangle_{\mathcal{M}}$, respectively) within a uniformly defined isophotal radius that encompasses almost their total emission.

It is motivated by the question of whether CBs and PBs are truly evolutionary distinct, or rather the opposite ends of a continuous sequence reflecting a decreasing relative contribution of "quick and early" to "slow and secular" processes to the bulge stellar mass growth. If so, in which manner might the 
relative importance of these two processes be imprinted on the evolutionary and chemical properties of bulges in present-day LTGs, and can it be linked to a semi-empirical indicator that could ease bulge classification? Another goal of this study is to explore through standard emission-line diagnostics the connection between gas excitation mechanisms and evolutionary properties of LTG bulges in order to gain insights into the occurrence of accretion-powered activity and its possible regulatory role on bulge growth.

To address these questions we extracted 135 LTGs from the CALIFA IFS survey (Sánchez et al. 2012, 2016) which were analyzed using spectral synthesis models and SDSS surface photometry. In Sect. 2, we describe the sample selection and the methodology employed for the spectral modeling and structural analysis of the galaxy sample. Section 3 provides an overview of the main results from this analysis, with a discussion following in Sect. 4. The conclusions from this study are summarized in Sect. 5.

\section{Data sample and methodology}

\subsection{Sample selection}

The galaxy sample analyzed here was extracted from the third data release of the CALIFA integral field spectroscopy (IFS) survey (667 galaxies; Sánchez et al. 2016) ${ }^{1}$, and comprises 135 non-interacting, nearly face-on local ( $\leq 130 \mathrm{Mpc})$ LTGs, selected for spatially resolved analysis of the physical and evolutionary properties of their bulge component. Systems strongly overlapping with bright foreground Galactic stars or extended background sources, or show signs of recent or ongoing interactions, or other morphological distortions (e.g., polar rings) were excluded. This is also the case for higher-inclination $\left(>40^{\circ}\right)$ LTGs in order to minimize internal obscuration effects and disk contamination. The sample may be considered representative for LTGs in the local universe, as it spans a range between -17.8 and -22.6 mag in SDSS $r$ absolute magnitude $M_{r}$ (Fig. 1), as determined from integration of SBPs down to an extinction-corrected surface brightness level $24 \mathrm{mag} / \square^{\prime \prime}$, and covers all late-type morphological types. With regard to the bulge component, the LTGs under study span a factor $\sim 250$ in optical luminosity $\left(-20.6 \lesssim M_{r}(\mathrm{mag}) \lessgtr-14.6\right)$ and $3 \mathrm{dex}$ in $\mathcal{M}_{\star}\left(10^{8.3}-10^{11.3} M_{\odot}\right.$; cf. Table C.1 and Fig. 6).

\subsection{Data analysis}

The methodology adopted in this study combines spatially resolved modeling of IFS data with two further elements. The first one is the structural analysis of SDSS $r$-band images in order to obtain a uniformly defined and largely model-independent isophotal radius for the bulge, within which physical and evolutionary quantities from spectral synthesis were subsequently analyzed. This ensures a homogeneous extraction and spectral fitting of nearly the total bulge emission, free of aperture biases that are inherent to single-fiber spectroscopy. The second novel element of our study is the spaxel-by-spaxel post-processing of the spectral synthesis output with the code REMOVEYOUNG ( $\mathcal{Y}$; Gomes \& Papaderos 2016) with the goal of a quantitative study of the contribution of stellar populations forming over the past 9 Gyr ( $\sim 2 / 3$ of the age of the Universe) to the optical surface brightness $(\mu)$ and $\Sigma_{\star}$ of the bulge and disk. The insights gained from this computationally expensive task, which is performed for the first time on a large set of IFS data here, will be discussed in detail in a forthcoming article.

\footnotetext{
1 See http://califa.caha.es
}

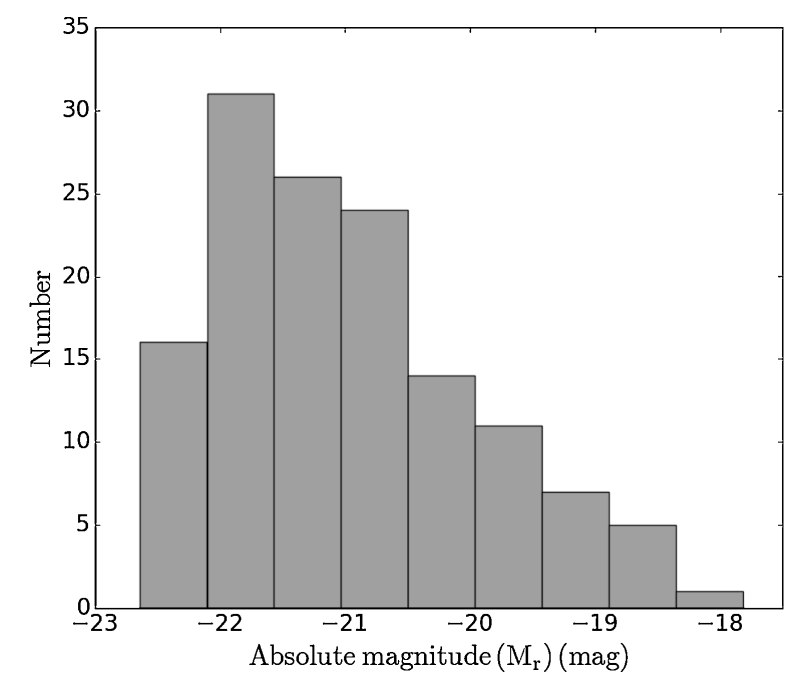

Fig. 1. Distribution of extinction-corrected total absolute magnitudes in the SDSS $r$ for the analyzed sample of 135 LTGs.

This pilot analysis revolves around $\left\langle\delta \mu_{9 \mathrm{G}}\right\rangle$, a new distanceindependent and, formally, also extinction-independent quantity obtained with $\mathcal{R} \mathcal{Y}$, which, as we discuss next, offers a semiempirical tracer of the physical and evolutionary properties of LTG bulges and possibly a convenient means for their classification.

\subsubsection{Determination of the bulge radius}

The bulge radius $R_{\mathrm{B}}$ was determined at an extinction-corrected surface brightness level $\mu_{\text {lim }}$ of $24 \mathrm{mag} / \square^{\prime \prime}$ by fitting a Sérsic model to the interactively selected central luminosity excess of SDSS $r$ SBPs with our surface photometry code iFit (Breda et al., in prep.). Additionally, a full image decomposition into bulge and disk, and whenever necessary a bar, was carried out with iFit, IMFIT (Erwin 2015) and GALFIT (Peng et al. 2010) in order to estimate the dependence of $R_{\mathrm{B}}$ on different codes and profile fitting schemes (see Fig. 2 for an illustrative example). The latter approach was found to yield a reduction of the radius of the bulge by on average $8 \%$, thereby leading to only small differences $(\lesssim 10 \%)$ in the quantities inferred for it from spectral modeling (cf. Sect. 2.2.2), as compared to those within the $R_{\mathrm{B}}$ from single Sérsic fits. Given the mean bulge diameter of $12^{\prime \prime} 2 \pm 4^{\prime \prime}$ in our sample, and based on simulations, it was found that profile smearing with the typical point spread function (PSF) of SDSS $r$-band data $\left(\sim 1\right.$ '. 3 ) has a negligible effect on $R_{\mathrm{B}}$ determinations.

In the following analysis, we refrain from a preliminary photometric subdivision of LTG bulges into CBs and PBs on the basis of the Sérsic index $\eta$, given its unclear physical meaning (cf. Sect. 1). This is also because the best-fitting Sérsic model parameters are sensitive to the details of the SBP decomposition, in particular on the modeling and subtraction of the underlying disk (e.g., Papaderos et al. 1996a; Noeske et al. 2003), and eventually the bar (Méndez-Abreu et al. 2008; Breda 2014). This is illustrated on the example of NGC 0776 (Fig. 2): it can be seen that automated fitting of a pure exponential to the down-bending (type ii) profile of the disk would overestimate its central surface brightness and underestimate its scale length $\alpha$. This would then lead to an underestimation of the excess emission from the bulge, and a false determination of its total magnitude, isophotal radius and Sérsic parameters. A meaningful approach in this case would be, either to model the disk with a modified exponential distribution (for example, the fitting function proposed in 


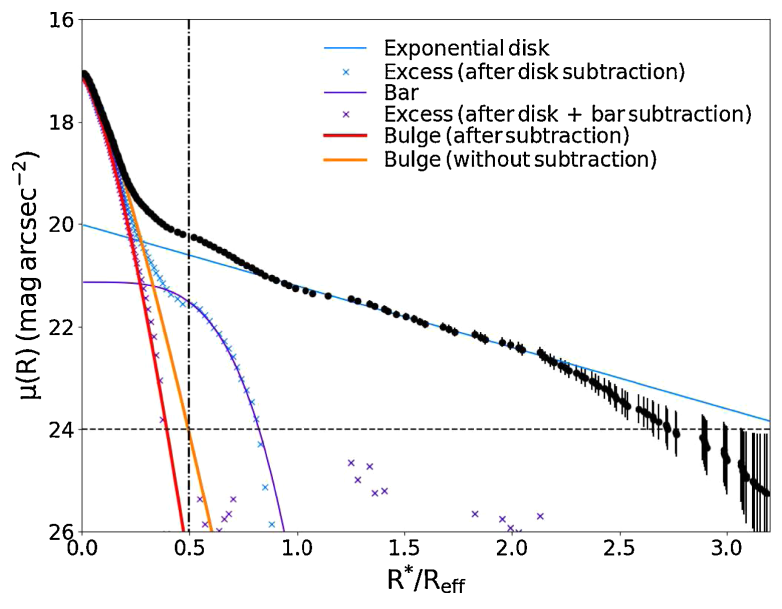

Fig. 2. Illustrative example of the decomposition with iFit of the SDSS $r$-band SBP of the LTG NGC 0776 (cf. Fig. 5) into bulge, bar and disk (red, magenta and blue, respectively). The bulge and bar are approximated by a Sérsic model, and the down-bending (type ii) disk by a pure exponential fitted to intermediate radii $\left(1 \leq R^{\star} / R_{\text {eff }} \leq 2.1\right.$, where $R_{\text {eff }}$ denotes the radius enclosing $50 \%$ of the total galaxy luminosity). The thick-orange curve shows a single Sérsic fit to the central luminosity excess owing to the bulge. It can be seen that its isophotal radius $R_{\mathrm{B}}$ at $\mu=24 \mathrm{mag} / \square^{\prime \prime}$ (dashed-dotted vertical line) determined from a singleSérsic model closely matches the one read off the figure for the case of full bulge-bar-disk decomposition.

Papaderos et al. 1996a), or to interactively select and fit the inner exponential part of the disk $\left(1 \leq R^{\star} / R_{\mathrm{eff}} \leq 2.1\right)$, as was done here (light-blue line in Fig. 2). Another salient feature of the SBP of NGC 0776 is a weak bump at $20 \lesssim \mu\left(r\right.$ mag/ $\left.\square^{\prime \prime}\right) \lesssim 21$ that reflects the emission from a bar, being well visible on the SDSS truecolor image composite (lower-left panel of Fig. 5); its neglect in 1D/2D decomposition could systematically impact Sérsic fits to the excess emission above the disk (open blue crosses), which encompasses the more extended lower-surface brightness (LSB) "wings" from the bar. As pointed out in Breda (2014), this could lead to an overestimation of $\eta$ and possibly move a PB into the locus of $\mathrm{CBs}$, eventually also increase the scatter in any relation between $\eta$ and other galaxy parameters (for example, bulge $\sigma_{\star}, M_{r}$, or mean surface brightness). With these considerations in mind, and to ensure that the evolutionary and spectroscopic properties of bulges (cf. Sects. 2.2.2 and 2.3, respectively) are obtained within a radius based on a uniform, clear-cut definition and without strong prior assumptions on the photometric structure of LTGs, we took the simpler approach of determining $R_{\mathrm{B}}$ from fitting a single Sérsic model to the central luminosity peak of the bulge upon visual inspection of the morphology and $g-i$ color maps of our LTG sample.

\subsubsection{Spectral modeling of CALIFA IFS data}

Spectral modeling of low resolution $(\lambda / \Delta \lambda \sim 6.5$ at $\sim 5000 \AA)$ CALIFA IFS data taken with the Potsdam Multi-Aperture Spectrometer (PMAS; Roth et al. 2005) in its PPaK mode (Verheijen et al. 2004; Kelz et al. 2006) with the V500 grating, and reduced as described in García-Benito et al. (2015, and references therein) was carried out with our pipeline PORTO3D (Papaderos et al. 2013; Gomes et al. 2016a, for details). Spectral fits were computed with the population spectral synthesis (PSS) code STARLIGHT (Cid Fernandes et al. 2005) in the spectral range between 4000 and $6800 \AA$ using a library of 152 simple stellar population (SSP) spectra. This library (hereafter Z4) comprises SSPs from Bruzual \& Charlot (2003) for 38 ages between
$1 \mathrm{Myr}$ and $13 \mathrm{Gyr}$ for four stellar metallicities (0.05, 0.2, 0.4 and $1.0 Z_{\odot}$ ), referring to a Salpeter initial mass function and Padova 2000 tracks. In order to evaluate the robustness of the results, the PSS modeling was repeated for a subset of the data using combined V500 and V1200 (COMB) ${ }^{2}$ CALIFA data covering the spectral range between 3700 and $7300 \AA$. These runs yielded differences of $\lesssim 0.2$ dex in $\mathcal{M}_{\star}$, that is within the typical uncertainties expected from PSS modeling with STARLIGHT (Cid Fernandes et al. 2014).

Additionally, the spaxel-by-spaxel modeling was repeated in the spectral range between 3900 and $6900 \AA$ with a SSP library that is identical to Z4 in terms of age coverage except for being supplemented by SSPs with a metallicity of $1.5 Z_{\odot}$ (hereafter Z5; 190 elements). A comparison of the results obtained with the Z5 and Z4 SSP base has shown that the global trends between $\left\langle\delta \mu_{9 \mathrm{G}}\right\rangle$ and other quantities considered in this study (stellar mass, surface density, age and metallicity; cf. Fig. 6) remain unaltered albeit systematic differences between individual determinations, which presumably reflect the age-metallicity degeneracy (AMD; Worthey et al. 1994). As expected, the Z5-based analysis yields a super-solar metallicity (up to $\sim 1.5 Z_{\odot}$ ) for high- $\mathcal{M}_{\star}$ bulges at a simultaneous reduction (by up to $\sim 2 \mathrm{Gyr}$ ) of $\left\langle t_{\star}\right\rangle_{\mathcal{M}}$, whereby differences in $\mathcal{M}_{\star}$ and $\Sigma_{\star}$ typically do not exceed $0.2-0.3$ dex. As for $\left\langle\delta \mu_{9 \mathrm{G}}\right\rangle$ (Sect. 2.2.3) the difference between Z5- and Z4based determinations is rather small $( \pm 0.5 \mathrm{mag})$ yet systematic, with a weak tendency for a decrease of $\left\langle\delta \mu_{9 \mathrm{G}}\right\rangle$ in high- $\mathcal{M}_{\star}$ bulges and vice versa. Notwithstanding this fact, the overall robustness of $\left\langle\delta \mu_{9 \mathrm{G}}\right\rangle$ and of its correlation with other properties, despite the notorious AMD, is reassuring and underscores its significant potential as a handy proxy of the physical and evolutionary characteristics of LTG bulges.

Whereas the main results from the Z5-based analysis are supplied in Fig. B.1 for the disposal of the reader and the sake of completeness, we adopt in the following the determinations based on the Z4 SSP library. Even though this choice might entail a potential saturation of $\left\langle Z_{\star}\right\rangle_{\mathcal{M}}$ at $Z_{\odot}$, it may be expected that, in the presence of the AMD, a narrowing-down of the SSP metallicity space has the advantage of tighter estimates on $\left\langle t_{\star}\right\rangle_{\mathcal{M}}$, which is the main focus of this study. Aside from that, it might be conjectured that the occupancy of the full available metallicity space in Z5 fits is partly driven by the mathematical/numerical foundation of state-of-the-art PSS codes, thus in itself no compelling evidence for a significantly oversolar mass-weighted stellar metallicity. For instance, whereas the light-weighted $\left\langle Z_{\star}\right\rangle_{\mathcal{L}}$ reflects the young and presumably more metal-enriched stellar component (in agreement with supersolar determinations for massive galaxy spheroids from, for example, luminosity-weighted Lick indices), the mass-weighted $\left\langle Z_{\star}\right\rangle_{\mathcal{M}}$ primarily reflects the older, high mass-to-light ratio stellar component that likely has had less time for its chemical self-enrichment. An inequality $\left\langle Z_{\star}\right\rangle_{\mathcal{M}} \leq\left\langle Z_{\star}\right\rangle_{\mathcal{L}}$ appears therefore conceivable from the evolutionary point of view. More generally, it should be kept in mind that the AMD and other potential sources of degeneracy (e.g., between metallicity and $\sigma_{\star}$, cf. Koleva et al. 2008) within the complex topology of non-linearly coupled parameters in current PSS models have not been fully addressed so far, which makes a conservative limitation of the $Z_{\star}$ parameter space an admissible option.

PORTO3D computes several quantities of interest, including the present-day and ever formed $\mathcal{M}_{\star}\left(M_{\odot}\right),\left\langle t_{\star}\right\rangle_{\mathcal{M}}(\mathrm{Gyr})$

\footnotetext{
2 We note that CALIFA DR 3 provides COMB data for 97 galaxies from our sample.
} 


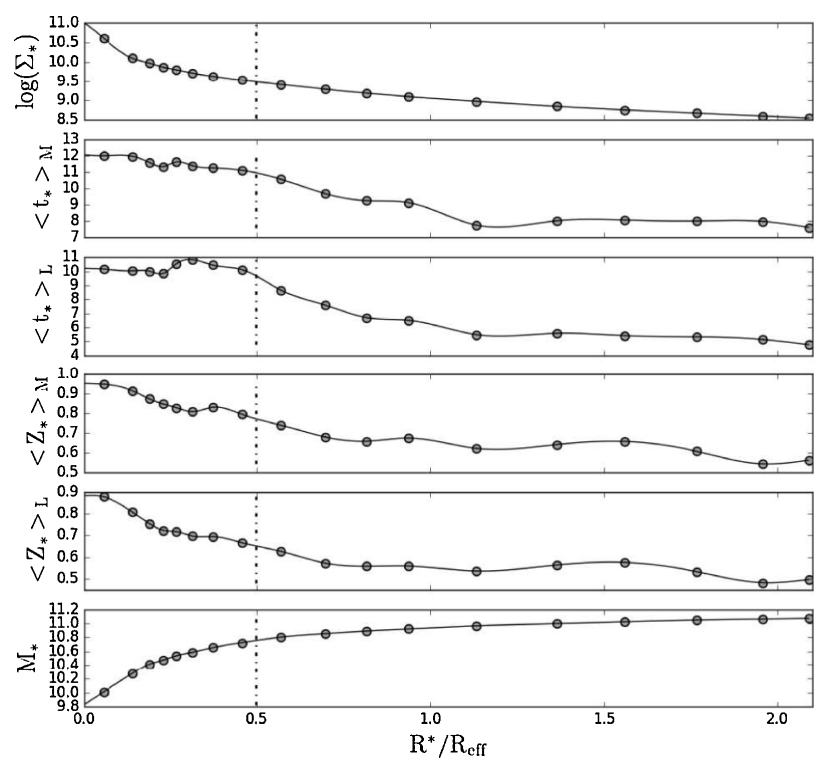

Fig. 3. Radial profiles for various quantities obtained for NGC 0776 with the isan technique and auxiliary codes for spline interpolation and statistical analysis. From top to bottom the panels show: $i$ ) a logarithmic representation of the stellar surface density $\Sigma_{\star}$ in $M_{\odot} / \mathrm{kpc}^{2}$, ii) and iii) the mass- and light-weighted stellar age $\left(\left\langle t_{\star}\right\rangle_{\mathcal{M}}\right.$ and $\left\langle t_{\star}\right\rangle_{\mathcal{L}}$, respectively) in Gyr, $i v)$ and $v)$ the mass- and light-weighted stellar metallicity $\left(\left\langle Z_{\star}\right\rangle_{\mathcal{M}}\right.$ and $\left\langle Z_{\star}\right\rangle_{\mathcal{L}}$, respectively) in $Z_{\odot}$, and $v i$ ) the enclosed present-day stellar mass $\mathcal{M}_{\star}$ as a function of the photometric radius $R^{\star}$ normalized to the effective radius $R_{\text {eff }}$. The bulge radius $R_{\mathrm{B}}$ is depicted by the vertical dashed line.

and metallicity $\left\langle Z_{\star}\right\rangle_{\mathcal{M}}\left(Z_{\odot}\right)$, and their luminosity-weighted values $\left(\left\langle t_{\star}\right\rangle_{\mathcal{L}}\right.$ and $\left.\left\langle Z_{\star}\right\rangle_{\mathcal{L}}\right)$, the stellar surface density $\Sigma_{\star}\left(M_{\odot} / \mathrm{kpc}^{2}\right)$, the time $t_{1 / 2}$ when $50 \%$ of the present-day $\mathcal{M}_{\star}$ was in place, the light and mass-fraction of stellar populations younger than $0.1,1$ and $5 \mathrm{Gyr}$, as well as emission-line fluxes and equivalent widths (EWs), and stellar and ionized-gas velocity maps (see Gomes et al. 2016a, for details). Here we limit the discussion to massweighted values, since they are robust against young $(\lessgtr 0.1 \mathrm{Gyr})$ stellar populations that typically dominate the light despite their very low $\mathcal{M}_{\star}$ fraction.

Spatially resolved maps of the aforementioned quantities were in turn converted into radial profiles using an adaptation of the isophotal annuli (isan) surface photometry technique by Papaderos et al. (2002). The key feature of this method (e.g., Kehrig et al. 2012; Papaderos et al. 2013; Gomes et al. 2016a) consists in the computation of statistics within logarithmically equidistant isophotal zones defined from a reference image in this case, the emission-line-free pseudo-continuum between 6390 and $6490 \AA$, segmented into 18 isophotal zones. The latter closely trace the galaxy morphology at all surface brightness levels, without the prior assumptions on galaxy structure commonly made in 1D/2D surface photometry techniques (approximation of a galaxy as due to superposition of axis-symmetric components, or profile derivation within elliptical annuli with constant ellipticity and position angle), this way permitting accurate determination of SBPs and color profiles for irregular galaxies (see also, e.g., Noeske et al. 2003). Figure 3 shows an example of radial profiles, after normalization ${ }^{3}$ to $R_{\text {eff }}$.

3 We note that the normalization of radial profiles in Fig. 3 to $r_{\mathrm{n}}=R^{\star} / R_{\mathrm{eff}}$ is for the sake of illustration only. Such a normalization is omitted in the forthcoming discussion because it could bias studies of the metallicity and star formation history (SFH) of the bulge and disk
In turn, isan determinations were spline-interpolated to a finer radius step to ensure that average values within $R_{\mathrm{B}}$ are not biased toward a group of data points that are eventually densely spaced in radius. The bulge mean stellar age $\left\langle t_{\star, B}\right\rangle_{\mathcal{M}}$ and metallicity $\left\langle Z_{\star, B}\right\rangle_{\mathcal{M}}$ were obtained as the arithmetic mean of these values, and the present-day stellar mass $M_{\star, \mathrm{B}}$ of the bulge was computed by integrating $\Sigma_{\star}$ profiles out to $R_{\mathrm{B}}$. Additionally, mean values for the bulge were computed by performing statistics directly on 2D maps within the isophote corresponding to $R_{\mathrm{B}}$, finding overall a satisfactory agreement with the previous determinations.

Even though all quantities above are weighted by $\mathcal{M}_{\star}$, being therefore relatively insensitive to the luminosity contribution by the star-forming disk, the fact that $R_{\mathrm{B}}$ extends in some cases (e.g., NGC 0776 in Fig. 2) far into the latter, and the bulge lineof-sight contribution sharply decreases beyond the bulge $R_{\text {eff }}$, calls for an evaluation of a possible contamination by the disk. A correction for the latter would in principle be possible, if its properties $\left(\Sigma_{\star},\left\langle t_{\star}\right\rangle_{\mathcal{M}}\right.$ and $\left.\left\langle Z_{\star}\right\rangle_{\mathcal{M}}\right)$ beneath the bulge could be constrained with sufficient accuracy, or at least coarsely from their estimated luminosity fraction within $R_{\mathrm{B}}$, or using hybrid spectro-photometric decomposition techniques (e.g., Johnston et al. 2017). These approaches, however, rely by necessity on simplifying assumptions on the photometric structure of LTGs, the most important of which being that the disk preserves its exponential slope at $R^{\star}>R_{\mathrm{B}}$ all the way to its center, and has throughout a zero radial gradient in age, mass-to-light ratio and metallicity. As these assumptions are controversial or incompatible to observations (e.g., Papaderos et al. 1996a; Noeske et al. 2003; Sánchez-Blázquez et al. 2014; Tissera et al. 2016), no attempt was made for a spectrophotometric disk subtraction, consistently with the approach taken in the photometric analysis (Sect. 2.2.1). However, a series of tests made within the central portion $\left(R^{\star} \leq 3^{\prime \prime}\right.$. 3$)$ of bulges, where contamination by the disk is minimal, has shown that mass-weighted quantities inferred therein are in good agreement with those within $R_{\mathrm{B}}$ (cf. Fig. A.1). This indicates that the luminosity contribution by the star-forming disk does not appreciably impact determinations of $\left\langle t_{\star, B}\right\rangle_{\mathcal{M}}$ and $\left\langle Z_{\star, B}\right\rangle_{\mathcal{M}}$ in Fig. 6 .

\subsubsection{Post-processing of the spectral synthesis output with REMOVEYOUNG}

RemoveYoung ( $\mathcal{R} \boldsymbol{Y}$; Gomes \& Papaderos 2016) is a tool intended to the post-processing of the population vector (PV; i.e., the best-fitting combination of fractional contributions of individual SSPs to the galaxy mass) obtained by modeling a spectrum with a PSS code (e.g., STARLIGHT in this case). $\mathcal{R} \mathcal{Y}$ permits removal from a PV of the contribution from SSPs younger than an adjustable age cutoff $t_{\text {cut }}$ and computation of the spectrum, magnitudes in different filters (e.g., SDSS $u, g$, $r, i, z)$ and stellar mass of the residual older stellar component. In particular, spaxel-by-spaxel application of $\mathcal{R} \mathcal{Y}$ to the spectral synthesis output from PORTO3D allows to strip off IFS data

component of LTGs. This is because $r_{\mathrm{n}}$ is coupled with the $\mathrm{B} / \mathrm{T}$ ratio: for instance, from Fig. 8 of Papaderos et al. (2006) it follows that the $R_{\text {eff }}$ of a bulgeless LTG corresponds to 1.7 exponential scale lengths $\alpha$ of the disk, whereas in a bulge dominated LTG with a $B / T=3 / 4$ the $R_{\text {eff }}$ shrinks to $\sim 0.5 \alpha$. Therefore, stacking or inter-comparison of $r_{\mathrm{n}}$-normalized profiles in an attempt to systematize radial trends in, for example, age, SFR and specific SFR (sSFR) in the bulge and disk of LTGs can lead to averaging of determinations within the bulge component of higher-B/T LTGs with those in the disk component of lower-B/T LTGs. 


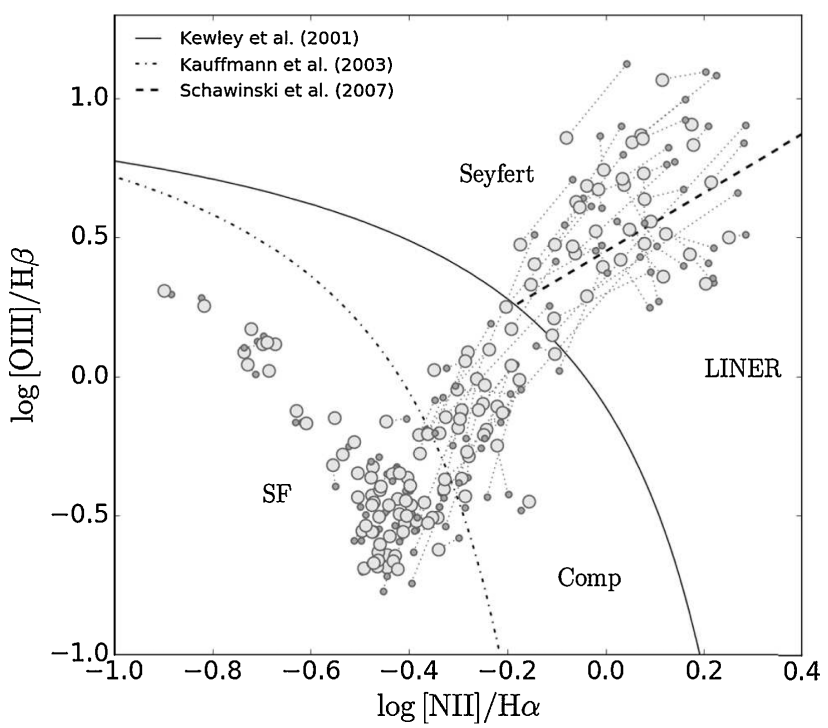

Fig. 4. Comparison of diagnostic emission-line ratios after Baldwin et al. (1981), as obtained within a $3^{\prime \prime}$ aperture (small circles; method a), connected by dotted lines with determinations from method $\mathrm{b}$ (mean ratios within $R_{\mathrm{B}}$ from radial [OIII]/H $\beta$ and [NII]/H $\alpha$ profiles; big circles). Data based on method c (luminosity-weighted determinations using integral [OIII]5007, $\mathrm{H} \beta,[\mathrm{NII}] 6584$ and $\mathrm{H} \alpha$ line fluxes within $R_{\mathrm{B}}$ ) and error bars are omitted for the sake of better visibility.The loci of Seyfert, LINERs and Composites, and that corresponding to photoionization by SF are demarcated following Kauffmann et al. (2003b, dashed-dotted curve), Kewley et al. (2001, solid curve) and Schawinski et al. (2007, dashed line).

cubes from, for example, the young ionizing stellar component $\left(t_{\mathrm{cut}} \sim 30 \mathrm{Myr}\right)$ and produce synthetic images of the underlying older stellar background in various photometric bands $(\mathcal{R} y$ convolves the residual spectral energy distribution (SED) with the filter transmission functions). In the framework of this study, $\mathcal{R} y$ was applied spaxel-by-spaxel to the LTG sample for eight $t_{\text {cut }}$ values $\left(0.03,0.1,0.3,1,3,5,7\right.$ and 9 Gyr). The $\mu$ and $\Sigma_{\star}$ maps from $\mathcal{R} \mathcal{Y}$ were in turn converted into 1D radial profiles as described in Sect. 2.2.2.

Figure 5 illustrates the application of $\mathcal{R} \mathcal{Y}$ on three LTGs from our sample. The synthetic $r$-band SBPs for the eight adopted age cutoffs are shown color coded. SBPs labeled " $r$ SDSS" were obtained for a $t_{\text {cut }}=0 \mathrm{Gyr}$, that is, through convolution of the observed IFS data cubes with the SDSS $r$-band transmission curve. For comparison, we overlay the $r$ band SBPs computed directly from SDSS images (light-gray curves labeled "OBS $r$ SDSS"), which due to their better resolution ( $F W H M \sim 1$.' 3 as compared to $\sim 2$ ". 6 for CALIFA IFS data) better trace the central luminosity peak of the bulge. It can be seen that whereas all three LTGs show a strong surface brightness dimming with increasing $t_{\text {cut }}$ in their disks, this is not necessarily the case for their bulge component (vertical gray line). For example, suppression of stellar populations of a successively higher age results in a roughly uniform dimming both in the disk and bulge of IC 0776, whereas it has practically no effect on the bulge surface brightness of NGC 0776 .

Based on the output from $\mathcal{R} \mathcal{Y}$, we computed the difference $\mu_{0 \mathrm{Gyr}}-\mu_{9 \mathrm{Gyr}}$ where $\mu_{0 \mathrm{Gyr}}$ and $\mu_{9 \mathrm{Gyr}}$ denote, respectively, the synthetic $r$-band SBP of each LTG for a $t_{\text {cut }}$ of 0 and 9 Gyr. The arithmetic average of this profile within $R_{\mathrm{B}}$ is referred to in the following as $\left\langle\delta \mu_{9 \mathrm{G}}\right\rangle$ (mag). For instance, a $\left\langle\delta \mu_{9 \mathrm{G}}\right\rangle=0 \mathrm{mag}$ corresponds to the case where stellar populations with age $\geq 9$ Gyr entirely dominate the $r$-band surface brightness within
$R_{\mathrm{B}}$, whereas a $\left\langle\delta \mu_{9 \mathrm{G}}\right\rangle$ of -2.5 mag translates into a contribution of $10 \%$ by this old stellar component. Even though a correlation between $\left\langle\delta \mu_{9 \mathrm{G}}\right\rangle$ and broadband colors appears plausible, it should be born in mind that these two quantities have a different definition, and the former (as well as any other quantity comparing $\mu$ for two different $t_{\text {cut }}$ 's, for example, 0.3 and $1 \mathrm{Gyr}$ ) yields a stronger age diagnostic, since, at variance to colors, in principle permits complete suppression of stellar populations younger than a given age. As we shall argue next, $\left\langle\delta \mu_{9 \mathrm{G}}\right\rangle$, introduced and analyzed for the first time here, offers a simple, distance- and formally extinction-independent proxy to the evolutionary and physical properties of LTG bulges ${ }^{4}$.

\subsection{Spectroscopic classification of LTG bulges}

Another aim of this study is the exploration of gas excitation mechanisms in LTG bulges across their relevant range in $\mathcal{M}_{\star}$ and $\Sigma_{\star}$ using a combination of methods that allow to quantify aperture biases in luminosity-weighted emission-line ratios. To this end, emission-line maps were determined by PORTO3D through spaxel-by-spaxel subtraction of the best-fitting stellar model from the input spectrum. The flux of the four emission lines ([OIII]5007, $\mathrm{H} \beta$, [NII]6584 and $\mathrm{H} \alpha$ ) used for spectroscopic classification after Baldwin et al. (1981, hereafter BPT) were then determined from stellar continuum-subtracted maps. An accurate starlight subtraction is crucial to the spectroscopic classification, inter alia because in areas with weak nebular emission, hydrogen Balmer lines embedded within broader stellar absorption profiles are eventually strongly underestimated when measured with standard line fitting techniques. As pointed out in Petropoulou et al. (2011), this could in turn artificially increase the $[\mathrm{OIII}] / \mathrm{H} \beta$ ratio, moving a source upward on BPT diagrams and eventually prompting its erroneous classification as a Seyfert.

BPT ratios for our LTG bulges were determined with three different methods. The first one (method a) simulates SDSS measurements within a $3^{\prime \prime}$ aperture centered on the maximum of the emission-line-free pseudo-continuum maps. This aperture diameter yields a good match to the angular resolution of CALIFA data and is generally much smaller than $R_{\mathrm{B}}$ (cf. Sect. 2.2.2 and Appendix A), thereby minimizing dilution of possible spectroscopic signatures from an Active Galactic Nucleus (AGN) by circumnuclear SF. Additionally, BPT ratios were determined by averaging out to $R_{\mathrm{B}}$ spline-interpolated [OIII] $/ \mathrm{H} \beta$ and $[\mathrm{NII}] / \mathrm{H} \alpha$ ratios within isan (method b), as well as from the integrated [OIII]5007, $\mathrm{H} \beta$, [NII]6584 and $\mathrm{H} \alpha$ line fluxes within $R_{\mathrm{B}}$ $(\operatorname{method} \mathrm{c})$. Whereas determinations with method a are most sensitive to a central AGN, the contrary is the case for the area-weighted BPT ratios from method $b$. This is because the latter are only weakly dependent on the luminosity contribution from a bright central point source, which, even if dominating the total nebular luminosity within the bulge, would affect radial $\log [\mathrm{OIII}] / \mathrm{H} \beta$ and $\log [\mathrm{NII}] / \mathrm{H} \alpha$ profiles only locally (out to $R^{\star} \sim$ FWHM), thereby having little influence on their mean value within $R_{\mathrm{B}}$. Method c, on the other hand, yields purely luminosity-weighted BPT ratios within $R_{\mathrm{B}}$ and simulates the (idealized) situation where the SDSS fiber precisely matches the isophotal bulge diameter of a LTG.

\footnotetext{
4 We note that the stellar extinction in the bulge component of our LTG sample is relatively low, with a mean value of $A_{V}=0.3 \pm 0.18 \mathrm{mag}$. $\left\langle\delta \mu_{9 \mathrm{G}}\right\rangle$ can be readily obtained by post-processing a PSS fit to any spectroscopic data set (e.g., single-fiber spectroscopy from the SDSS and GAMA surveys) with the publicly available version of $\mathcal{R} \mathcal{Y}$ (cf. wwW . iastro.pt/research/tools/RemoveYoung.html).
} 
Differences between method $\mathrm{b}$ and $\mathrm{c}$ were found to be generally small $(\lessgtr 0.3 \mathrm{dex})$ and in most cases compatible to those obtained with method a, therefore not globally altering the spectroscopic classification of the analyzed bulges, in particular for sources classified as SF. However, as apparent from the upperright part of Fig. 4, there is a tendency for method b (large circles) to move determinations within a $3^{\prime \prime}$ aperture (method a, small circles) from the locus of LINERs (Heckman 1980) and Seyferts into the locus of Composites.

This trend, which can be attributed to dilution of central AGN/LINER emission by circumnuclear SF, is in accord with the conjecture by Gomes et al. (2016b) that the upper-right "wing" delineated by SDSS galaxies on the BPT diagram is partly due to aperture effects and consistent with an inside-out galaxy formation (or, SF quenching) scenario. It is also interesting in this context that Iglésias-Páramo et al. (2016) document from analysis of CALIFA IFS data that integrated [OII] $/ \mathrm{H} \beta$ and $[\mathrm{NII}] / \mathrm{H} \alpha$ ratios for local LTGs can differ from single-fiber SDSS determinations by up to $\sim 0.3$ dex.

It is worth noting that, whereas Seyfert or Composite BPT ratios imply that an AGN dominates or substantially contributes to the gas excitation, the role of accretion-powered nuclear activity in bulges classified as LINERs is less clear. Traditionally, LINER emission-line ratios in massive, high- $\Sigma_{\star}$ spheroids (early-type galaxies and bulges) were ascribed to a diffuse floor of photoionization powered by the hard radiation field from hot evolved $\left(\geq 10^{8} \mathrm{yr}\right)$ post-asymptotic giant branch (pAGB) stars (e.g., Trinchieri \& di Serego Alighieri 1991; Binette et al. 1994; Macchetto et al. 1996; Stasińska et al. 2008; Cid Fernandes et al. 2010, 2011; Sarzi et al. 2010; Yan \& Blanton 2012). However, the pAGB photoionization hypothesis is valid only as long as the observed $\mathrm{EW}(\mathrm{H} \alpha)_{\text {obs }}$ does not exceed $\sim 3 \AA$, since this is the maximal, nearly metallicity-independent value predicted by zero-dimensional (OD) ESS models for an old, instantaneously formed stellar population, provided that case B recombination applies (e.g., Cid Fernandes et al. 2011; Gomes et al. 2016a). More specifically, as pointed out in Papaderos et al. (2013, hereafter P13), whereas an $\mathrm{EW}(\mathrm{H} \alpha)_{\mathrm{obs}} \leq 3 \AA$ is a necessary condition for the $\mathrm{pAGB}$ photoionization hypothesis to be tenable, it is in itself no compelling evidence against an $\mathrm{AGN}^{5}$.

Other interpretations for the origin of LINER emission involve fast shocks (e.g., Dopita \& Sutherland 1995; Allen et al. 2008) and gas excitation by a radiatively inefficient lowluminosity AGN (e.g., Ho 1999). P13 argue that even a strong AGN can not be ruled out in the LINER nuclei of many earlytype galaxies (ETGs). This is because the Lyman continuum $\left(\mathrm{Ly}_{\mathrm{c}}\right)$ photon escape fraction in the centers of these systems can reach values $\gtrsim 0.9$, which implies that the bulk of ionizing radiation from a putative AGN escapes without being locally reprocessed into nebular emission. $\mathrm{Ly}_{\mathrm{c}}$ photon escape, in conjunction with the EW dilution effect provides therefore an ansatz for understanding why many ETGs with clear evidence for a prodigious energetic output from an AGN (e.g., radio- and even

\footnotetext{
5 This is because in a triaxial geometry, dilution of the intrinsic (nuclear) $\mathrm{EW}(\mathrm{H} \alpha)_{\text {nuc }}$ by the stellar background along the line of sight implies an $\mathrm{EW}(\mathrm{H} \alpha)_{\text {nuc }} \geq \mathrm{EW}(\mathrm{H} \alpha)_{\text {obs }}$, with equality between these quantities representing a special case. Actually, the spatial anti-correlation between emission-line EWs and $\Sigma_{\star}$ observed in many star-forming galaxies nicely illustrates this effect (Papaderos et al. 2002), and together with the considerations above calls attention to the fact that interpreting projected observables and byproducts thereof (e.g., colors, EWs, Lick indices and ages, SFHs, metallicity enrichment histories, respectively) using predictions from standard 0D evolutionary synthesis models is not a straight forward task.
}

X-ray jets, as in the case of the LINER ETG M87 in the Virgo galaxy cluster) show weak, if any at all, nebular line emission. As conjectured in P13, the typically LINER BPT ratios in these massive galaxy spheroids may witness a situation where tenuous gas - permitting due to its very low density a high $\mathrm{Ly}_{\mathrm{c}}$ escape fraction - is exposed to the hard radiation field from an AGN and the diffuse post-AGB component. Indeed, the $\mathrm{Ly}_{\mathrm{c}}$ escape fraction is anti-correlated with $\mathrm{EW}(\mathrm{H} \alpha)$ (cf. their Fig. 2) and invariably exceeds 0.5 in LINER ETG nuclei. Evidently, the same association between LINER emission and extensive Lyc photon escape from a virtually gas-evacuated high- $\Sigma_{\star}$ stellar spheroid is also conceivable for massive LTG bulges hosting an AGN. On the basis of such considerations we do not exclude in the discussion in Sect. 4 that LTG bulges falling in the LINER locus of BPT diagrams could host significant accretion-powered nuclear activity.

\section{Results}

In this section, we provide an overview of the main results obtained, laying emphasis on the relation between $\left\langle\delta \mu_{9 \mathrm{G}}\right\rangle$ and the evolutionary and physical characteristics of LTG bulges in our sample. Additionally, we examine the variation of the bulgeto-disk age and metallicity contrast as a function of LTG mass, as well as the variation of the dominant gas excitation mechanisms along the bulge mass and age sequence.

\subsection{Physical and evolutionary properties of LTG bulges vs. $\left\langle\delta \mu_{9 \mathrm{G}}\right\rangle$}

Figure 5 illustrates three characteristic snapshots along a sequence of increasing galaxy $\mathcal{M}_{\star}$ and bulge $\left\langle\delta \mu_{9 \mathrm{G}}\right\rangle$ : whereas all three LTGs shown display a significant $(>1.5 \mathrm{mag})$ surface brightness dimming with increasing $t_{\text {cut }}$ in the disk $\left(R^{\star} \geq R_{\mathrm{B}}\right)$, implying its continued growth over the past $9 \mathrm{Gyr}$, their bulge $\left\langle\delta \mu_{9 \mathrm{G}}\right\rangle$ spans a broad range between $\sim 0 \mathrm{mag}$ and $\sim-4 \mathrm{mag}$, which translates, respectively, into a $r$-band luminosity fraction between $\sim 0 \%$ and $\sim 97 \%$ by stars younger than $9 \mathrm{Gyr}$.

A salient feature of almost bulgeless LTGs (e.g., IC 0776) with a $\left\langle\delta \mu_{9 \mathrm{G}}\right\rangle \leq-1.5 \mathrm{mag}$ (hereafter, $\left\langle\delta \mu_{9 \mathrm{G}}\right\rangle$ interval iA; 34 galaxies) is a roughly uniform dimming in $\mu$ with increasing $t_{\text {cut }}$ both in the bulge and the disk, which is consistent with a nearly homologous growth of $\Sigma_{\star}$ throughout the galaxy's extent. To the contrary, systems like NGC $0776\left(\left\langle\delta \mu_{9 \mathrm{G}}\right\rangle \geq-0.5 \mathrm{mag}\right.$; interval iC: 43 galaxies) show strong recent evolution only in their disks, whereas their $\left\langle\delta \mu_{9 \mathrm{G}}\right\rangle$ documents a dominant old stellar population in the bulge with no appreciable SF occurring therein over the past $9 \mathrm{Gyr}$. As for LTGs in the intermediate range of $\left\langle\delta \mu_{9 \mathrm{G}}\right\rangle$ $(-1.5$ to $-0.5 \mathrm{mag}$; subset iB: 58 galaxies; e.g., NGC 0001), spectral modeling points to a significant contribution from stars younger than 9 Gyr to the bulge luminosity.

A question next is, how the physical and evolutionary properties of LTG bulges may vary across these three tentatively defined intervals in $\left\langle\delta \mu_{9 \mathrm{G}}\right\rangle$.

A synopsis of the main results obtained for our sample is given in Fig. 6 (see also Table 1). Panel a reveals a nearly linear relation between $\left\langle\delta \mu_{9 \mathrm{G}}\right\rangle$ and mass-weighted stellar age $\left\langle t_{\star, \mathrm{B}}\right\rangle_{\mathcal{M}}$ for the bulge, with the transition from interval iA (blue dots) to interval iB (green dots) occurring at $\left\langle t_{\star, B}\right\rangle_{\mathcal{M}} \sim 9$ Gyr, and bulges in the interval iC (red dots) populating the upper-right part of the diagram $\left(\left\langle t_{\star, \mathrm{B}}\right\rangle_{\mathcal{M}} \gtrsim 11 \mathrm{Gyr}\right)$. These comparisons show that bulges have a large range in SSP-equivalent ages from $\sim 2$ to 13.5 Gyr (cf. e.g., Peletier et al. 2007; Moorthy \& Holtzman 2006) and metallicities. 
The tight trend between age and $\left\langle\delta \mu_{9 \mathrm{G}}\right\rangle$ in this panel can be approximated $^{6}$ by the relation $\left\langle t_{\star, \mathrm{B}}\right\rangle_{\mathcal{M}}(\mathrm{Gyr})=(12.14 \pm 0.06)+$ $(2.05 \pm 0.04) \cdot\left\langle\delta \mu_{9 \mathrm{G}}\right\rangle$ (solid line).

Panel $b$ shows that the age of LTG bulges (Gyr) roughly scales with the logarithm of their stellar mass $\left(M_{\odot}\right)$ as $\left\langle t_{\star, \mathrm{B}}\right\rangle_{\mathcal{M}}=(2.30 \pm 0.11) \cdot \log \mathcal{M}_{\star, \mathrm{B}}-(13.44 \pm 1.13)$. This implies that old $(\geq 11 \mathrm{Gyr})$ bulges in the $\left\langle\delta \mu_{9 \mathrm{G}}\right\rangle$ interval iC are by $\sim 2$ orders of magnitude more massive than bulges falling in the $\left\langle\delta \mu_{9 \mathrm{G}}\right\rangle$ interval $\mathrm{iA}$. The mean age of the latter was determined to be $6.82 \pm 1.06 \mathrm{Gyr}$, nearly $4 \mathrm{Gyr}$ lower than that of bulges in the interval ic $(10.7 \pm 0.4 \mathrm{Gyr})$, with bulges in the interval $i B$ having an average age of $9.80 \pm 0.7$ Gyr. As apparent from panels $\mathrm{d}$ and $\mathrm{e}$, massive old bulges (iC) are the most metal enriched and show the highest $\Sigma_{\star, \mathrm{B}}$, which in all cases exceeds $10^{9} M_{\odot} \mathrm{kpc}^{-2}$ and reaches up to $\sim 6 \times 10^{9} M_{\odot} \mathrm{kpc}^{-2}$, whereby the bulge stellar surface density scales as $\log \Sigma_{\star, \mathrm{B}}=(4.96 \pm 0.23)+(0.42 \pm 0.02) \cdot \log \mathcal{M}_{\star, \mathrm{B}}$ and $\log \Sigma_{\star, \mathrm{B}}=(7.63 \pm 0.09)+(0.16 \pm 0.01) \cdot\left\langle t_{\star, \mathrm{B}}\right\rangle_{\mathcal{M}}$.

From the combined evidence of panels a, b and e, a trend between $\left\langle\delta \mu_{9 \mathrm{G}}\right\rangle$ with bulge mass and surface density is to be expected. Indeed, linear fits to the data yield tight relations of the form $\log \mathcal{M}_{\star, \mathrm{B}}=(10.87 \pm 0.06)+(0.67 \pm 0.04) \cdot\left\langle\delta \mu_{9 \mathrm{G}}\right\rangle$ and $\log \Sigma_{\star, \mathrm{B}}=(9.56 \pm 0.03)+(0.32 \pm 0.02) \cdot\left\langle\delta \mu_{9 \mathrm{G}}\right\rangle$, suggesting that $\left\langle\delta \mu_{9 \mathrm{G}}\right\rangle$ offers a useful semi-empirical proxy to the evolutionary and physical properties of LTG bulges.

Finally, within the considered range of stellar metallicities $\left(Z_{\odot} / 20-Z_{\odot}\right)$, linear fits to $\left\langle Z_{\star, \mathrm{B}}\right\rangle_{\mathcal{M}}$ (panel d) yield the relations $\left\langle Z_{\star, \mathrm{B}}\right\rangle_{\mathcal{M}}\left(Z_{\odot}\right)=(0.23 \pm 0.01) \cdot \log \mathcal{M}_{\star, \mathrm{B}}-(1.60 \pm 0.11)$ and $\left\langle Z_{\star, \mathrm{B}}\right\rangle_{\mathcal{M}}\left(Z_{\odot}\right)=(0.28 \pm 0.02) \cdot \log \mathcal{M}_{\star, \mathrm{T}}-(2.37 \pm 0.15)$.

It is interesting to note that the age and metallicity of the disk $\left(R^{\star} \geq R_{\mathrm{B}}\right)$ follow similar relations with total stellar mass $\mathcal{M}_{\star, \mathrm{T}}$ $\left(M_{\odot}\right)$, as $\left\langle t_{\star, \mathrm{D}}\right\rangle_{\mathcal{M}}=(1.88 \pm 0.12) \cdot \log \mathcal{M}_{\star, \mathrm{T}}-(11.85 \pm 1.32)$ and $\left\langle Z_{\star, \mathrm{D}}\right\rangle_{\mathcal{M}}\left(Z_{\odot}\right)=(0.20 \pm 0.02) \cdot \log \mathcal{M}_{\star, \mathrm{T}}-(1.55 \pm 0.16)$.

Another issue of interest concerns the contribution of the bulge to the total LTG mass $\mathcal{M}_{\star, T}$. Panel c shows that more massive bulges are hosted by more massive (and luminous) LTGs, following a relation $\log \mathcal{M}_{\star, \mathrm{B}}=(1.22 \pm 0.02) \log \mathcal{M}_{\star, \mathrm{T}}$ $-(2.87 \pm 0.25)$, or, equivalently, $\mathcal{M}_{\star, \mathrm{B}} / \mathcal{M}_{\star, \mathrm{T}}=(0.13 \pm 0.02)$. $\log \mathcal{M}_{\star, T}-(1.12 \pm 0.17)$. The obtained relation (solid line) implies that bulges in the $\left\langle\delta \mu_{9 \mathrm{G}}\right\rangle$ interval iC contain on average $\sim 40 \%$ of the total stellar mass of a LTG, twice the mass fraction determined for bulges falling in the interval iA.

\subsection{Gas excitation mechanisms vs. bulge $\left\langle\delta \mu_{9 \mathrm{G}}\right\rangle$}

The importance of various gas excitation mechanisms in the three $\left\langle\delta \mu_{9 \mathrm{G}}\right\rangle$ intervals is examined in panel $\mathrm{i}$, on the basis of BPT diagnostics within simulated $3^{\prime \prime}$ SDSS apertures (method a in Sect. 2.3). It can be seen that accretion-powered nuclear activity (reflected in Seyfert- and eventually also LINER-specific BPT line ratios) does not manifest itself uniformly along the bulge $\left\langle\delta \mu_{9 \mathrm{G}}\right\rangle$ vs. $\mathcal{M}_{\star, \mathrm{B}}-\Sigma_{\star, \mathrm{B}}-\left\langle t_{\star, \mathrm{B}}\right\rangle_{\mathcal{M}}$ sequence, but is confined to the $\left\langle\delta \mu_{9 \mathrm{G}}\right\rangle$ interval $\mathrm{iB}-\mathrm{iC}$. Whereas this is consistent with the previously reported scarcity of Seyfert activity in intermediateto-low luminosity bulges that are commonly associated with PBs (Kormendy \& Ho 2013, for a review), an insight from this panel is the association between physical and evolutionary properties of bulges with their activity status: lower $\mathcal{M}_{\star, B}$ and $\Sigma_{\star, \mathrm{B}}$ iA bulges fall almost exclusively in the locus of SF,

\footnotetext{
6 Linear fits in this section and in Appendix B were computed through $\chi^{2}$ minimization for both quantities considered, given that none of them is strictly independent. Table B.1 additionally lists the coefficients from regression analysis minimizing $\chi^{2}$ along the abscissa only.
}
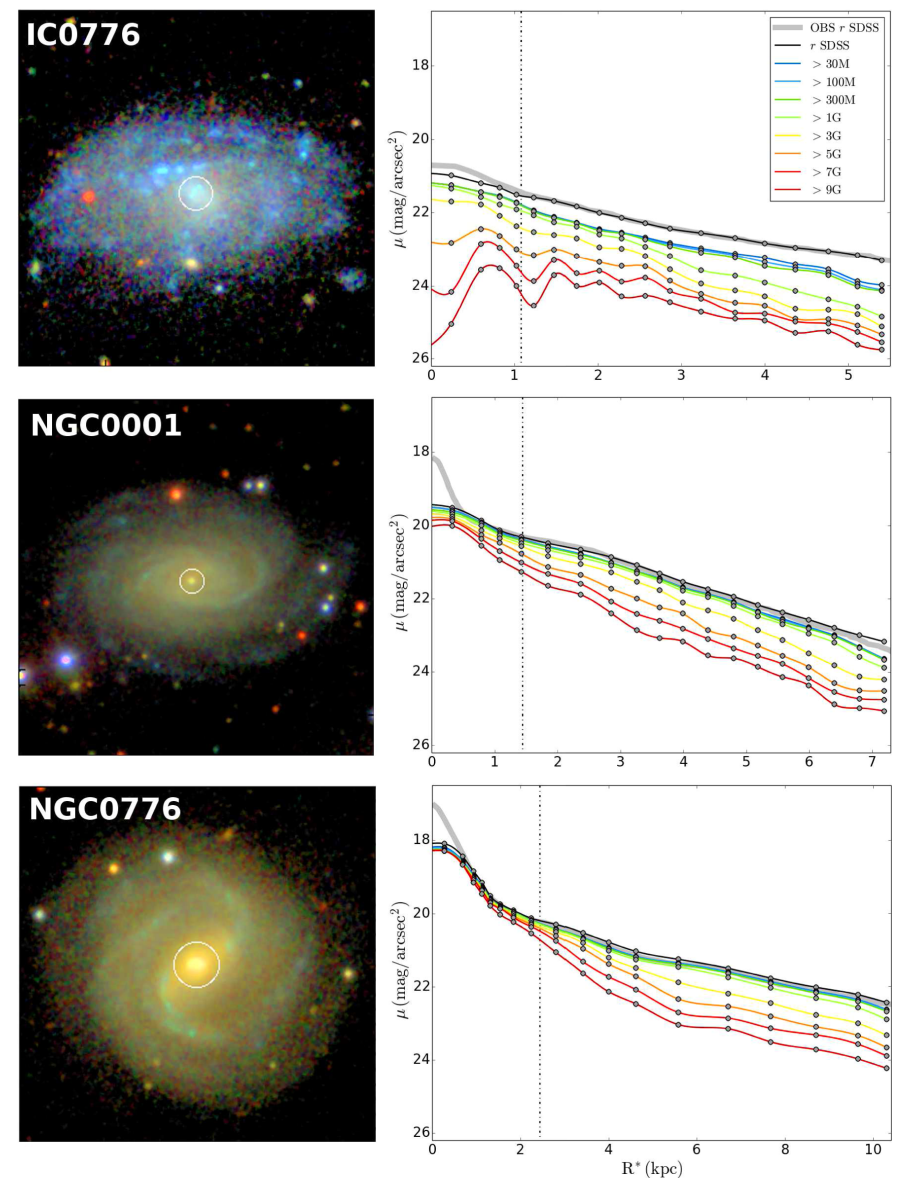

Fig. 5. SDSS true-color images and SBPs (left- and right-side panels, respectively) for three LTGs illustrating the prominence of the bulge relatively to the disk for the three $\left\langle\delta \mu_{9 \mathrm{G}}\right\rangle$ intervals tentatively defined in Sect. 3: IC $0776\left(\log \mathcal{M}_{\star, T}=9.58\right.$; interval iA), NGC $0001\left(\log \mathcal{M}_{\star, T}=\right.$ 10.99; interval iB) and NGC $0776\left(\log \mathcal{M}_{\star, T}=11.09\right.$; interval iC). Synthetic SBPs computed through convolution of the observed IFS data with the SDSS $r$-band filter transmission curve are shown in black, and those after removal with $\mathcal{R} \boldsymbol{Y}$ of stellar populations younger than 0.03 , $0.1,0.3,1,3,5,7$ and $9 \mathrm{Gyr}$ with the color coding in the upper-right panel. Thick-gray curves depict $r$-band SBPs computed from SDSS images and used for fitting a Sérsic model to infer the bulge radius $R_{\mathrm{B}}$ (dashed vertical lines). The circles overlaid with SDSS images depict the bulge diameter.

whereas higher-mass $\left(\gtrsim 10^{10} M_{\odot}\right)$, higher- $\Sigma_{\star, \mathrm{B}}\left(\gtrsim 10^{9} M_{\odot} \mathrm{kpc}^{-2}\right)$ iC bulges show in their majority Seyfert- and LINER-typical BPT ratios.

Quantitatively, $\sim 93 \%$ (38/41) of the bulges classified as Seyfert and LINER in our sample fall within the grayshaded quadrant in panel e that depicts the area $\left(\log \mathcal{M}_{\star, \mathrm{B}}\right.$; $\left.\log \Sigma_{\star, B}\right) \geq(10 ; 9)$. Of the 76 LTG bulges in this locus of the diagram, 16 are classified as Composite, reflecting gas excitation by a mixture of accretion-powered and SF activity. As already mentioned in Sect. 2.3, the proportion among different spectroscopic classes does not drastically change when area-weighted determinations within $R_{\mathrm{B}}$ (method b) are considered instead. These yield within the gray-shaded quadrant a proportion 23:11:22:20 between Seyfert, LINER, Composite and SF, lending further support to the conclusion that accretion-powered nuclear activity is primarily associated with old, high- $\mathcal{M}_{\star, \mathrm{B}}$ and high- $\Sigma_{\star, \mathrm{B}}$ bulges in the upper range of the $\left\langle\delta \mu_{9 \mathrm{G}}\right\rangle$ sequence. 

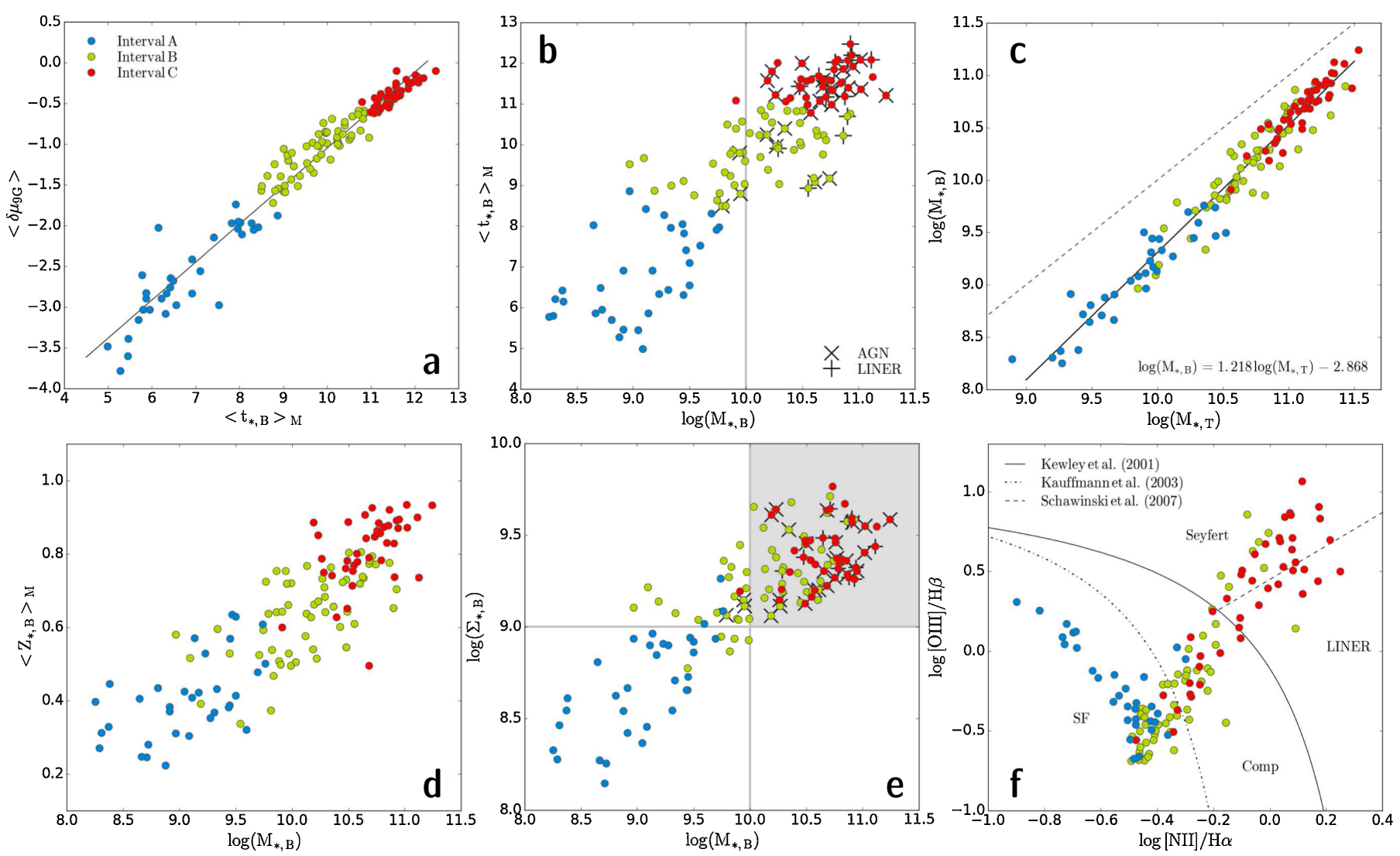

Fig. 6. Panel $a$ : $\left\langle\delta \mu_{9 \mathrm{G}}\right\rangle(r \mathrm{mag})$ vs. mass-weighted stellar age $\left\langle t_{\star, \mathrm{B}}\right\rangle_{\mathcal{M}}$ (Gyr) for the bulge component of our sample LTGs. The solid line shows a linear fit to the data. Panel $b$ : logarithm of the stellar mass $\mathcal{M}_{\star, \mathrm{B}}\left(M_{\odot}\right)$ in the bulge vs. mass-weighted stellar age $\left\langle t_{\star, \mathrm{B}}\right\rangle_{\mathcal{M}}(\mathrm{Gyr})$. Galaxies spectroscopically classified as Seyfert and LINER are marked. Panel $c$ : total stellar mass $\mathcal{M}_{\star, T}$ vs. $\mathcal{M}_{\star, \mathrm{B}}$. The solid and dashed lines show, respectively, a linear fit to the data and equality between both quantities. Panel $d$ : logarithm of $\mathcal{M}_{\star, \mathrm{B}}$ vs. mass-weighted stellar metallicity $\left\langle Z_{\star, \mathrm{B}}\right\rangle_{\mathcal{M}}\left(Z_{\odot}\right)$. Panel $e$ : logarithm of $\mathcal{M}_{\star, \mathrm{B}}$ vs. logarithm of the mean stellar surface density $\Sigma_{\star, \mathrm{B}}\left(M_{\odot} \mathrm{kpc}^{-2}\right)$ in the bulge. The gray-shaded quadrant at log $\mathcal{M}_{\star} \geq 10$ and $\log \Sigma_{\star, \mathrm{B}} \geq 9$ delineates the parameter space containing $93 \%$ of all Seyfert and LINER galaxies in our sample. Panel f: spectroscopic classification after BPT within simulated 3" SDSS fibers (method a in Sect. 2.3; cf. Fig. 4).

\subsection{Bulge-to-disk age and metallicity contrast}

A comparative study of the evolutionary properties of bulges relative to those of their hosting disks may add further insights into the bulge growth process. In this regard, it is noteworthy that all bulges in our sample contain a non-negligible mass fraction $m_{9}(\%)$ of stars older than $9 \mathrm{Gyr}$, with average values of $21 \pm 0.10,59 \pm 0.15$ and $85 \pm 0.07$ in the $\left\langle\delta \mu_{9 \mathrm{G}}\right\rangle$ intervals iA, iB and iC, respectively. The respective $m_{9}$ determinations for the disk $(24 \pm 0.09,36 \pm 0.08$ and $49 \pm 0.11)$ suggest that the oldest bulges are hosted by the oldest disks, and vice versa.

Indeed, a relation between the evolutionary properties of the disk $\left(R^{\star} \geq R_{\mathrm{B}}\right)$ and the bulge is suggested from panels $\mathrm{a}$ and $\mathrm{b}$ of Fig. 7, that show the mass-weighted age and metallicity of the bulge and disk (large and small dots, respectively) as a function of total stellar mass $\mathcal{M}_{\star, T}$. It can be appreciated from linear fits to the data (solid lines) that LTG disks follow similar yet shallower trends with $\mathcal{M}_{\star, T}$ as bulges, which is consistent with a synchronized evolution of the age and metallicity of the disk $\left(\left\langle t_{\star, \mathrm{D}}\right\rangle_{\mathcal{M}}\right.$ and $\left\langle Z_{\star, \mathrm{D}}\right\rangle_{\mathcal{M}}$, respectively) with $\left\langle t_{\star, \mathrm{B}}\right\rangle_{\mathcal{M}}$ and $\left\langle Z_{\star, \mathrm{B}}\right\rangle_{\mathcal{M}}$. Our data yield the relations $\left\langle t_{\star, \mathrm{B}}\right\rangle_{\mathcal{M}}=(1.21 \pm 0.06) \cdot\left\langle t_{\star, \mathrm{D}}\right\rangle_{\mathcal{M}}-(0.18 \pm$ $0.49)$ and $\left\langle Z_{\star, \mathrm{B}}\right\rangle_{\mathcal{M}}=(1.09 \pm 0.05) \cdot\left\langle Z_{\star, \mathrm{D}}\right\rangle_{\mathcal{M}}-(0.05 \pm 0.03)$.

As better visible from panel c, approximately all bulges are on average older than the disk (the exception are the lowest mass LTGs whose bulge and disk are almost indistinguishable from one another with respect to their mean age and metallicity). Interestingly, the bulge-to-disk age contrast $\delta t_{\mathrm{BD}}$ $\left(\left\langle t_{\star, \mathrm{B}}\right\rangle_{\mathcal{M}}-\left\langle t_{\star, \mathrm{D}}\right\rangle_{\mathcal{M}}\right)$ increases with $\mathcal{M}_{\star, \mathrm{T}}$ as $\delta t_{\mathrm{BD}}(\mathrm{Gyr})=(1.00 \pm$ $0.12) \cdot \log \mathcal{M}_{\star, \mathrm{T}}-(9.07 \pm 1.28)$. A similar, though weaker, trend can be appreciated from panel $\mathrm{d}$ for the bulge-to-disk metallicity contrast $\delta Z_{\mathrm{BD}}\left(\left\langle Z_{\star, \mathrm{B}}\right\rangle_{\mathcal{M}}-\left\langle Z_{\star, \mathrm{D}}\right\rangle_{\mathcal{M}}\right)$ that scales with galaxy mass as $\delta Z_{\mathrm{BD}}\left(Z_{\odot}\right)=(0.08 \pm 0.01) \cdot \log \mathcal{M}_{\star, \mathrm{T}}-(0.75 \pm 0.12)$. The mean $\delta t_{\mathrm{BD}}$ in the $\left\langle\delta \mu_{9 \mathrm{G}}\right\rangle$ intervals $\mathrm{iA}$, iB and iC was determined to be $0.50 \pm 1.1,1.65 \pm 0.7$ and $2.07 \pm 0.6 \mathrm{Gyr}$. It is also interesting to note that the age contrast in iC bulges shows a large spread between $\sim 0.5$ and $\sim 3.6 \mathrm{Gyr}$, and that in up to $\sim 40 \%$ of the bulges falling in the interval iA it is slightly negative ( $-1 \mathrm{Gyr})$, hinting at a rise of SF activity in the bulge over the recent few Gyr. As expected from the evidence of Fig. $6, \delta t_{\mathrm{BD}}$ and $\delta Z_{\mathrm{BD}}$ also show a positive correlation with $\left\langle\delta \mu_{9 \mathrm{G}}\right\rangle$.

Our conclusions above are in qualitative agreement with those by Sánchez-Blázquez et al. (2014) who find from a spectral modeling study of 62 nearly face-on spiral galaxies from CALIFA that bulges show, on average, higher luminosityweighted ages and metallicities than disks. The latter study also shows that the metallicities of bulge and disk are correlated (see also, e.g., Moorthy \& Holtzman 2006), in agreement with the trend in Fig. 7b, whereas no correlation was found between the age of the two components (see also, Sánchez-Blázquez 2016). Another insight from the study by Sánchez-Blázquez et al. (2014) is that the slope of the relation between central velocity dispersion and luminosity-weighted age and metallicity is similar for the central parts of bulges and at a galactocentric radius equivalent to $\sim 2.4$ exponential disk scale lengths. 

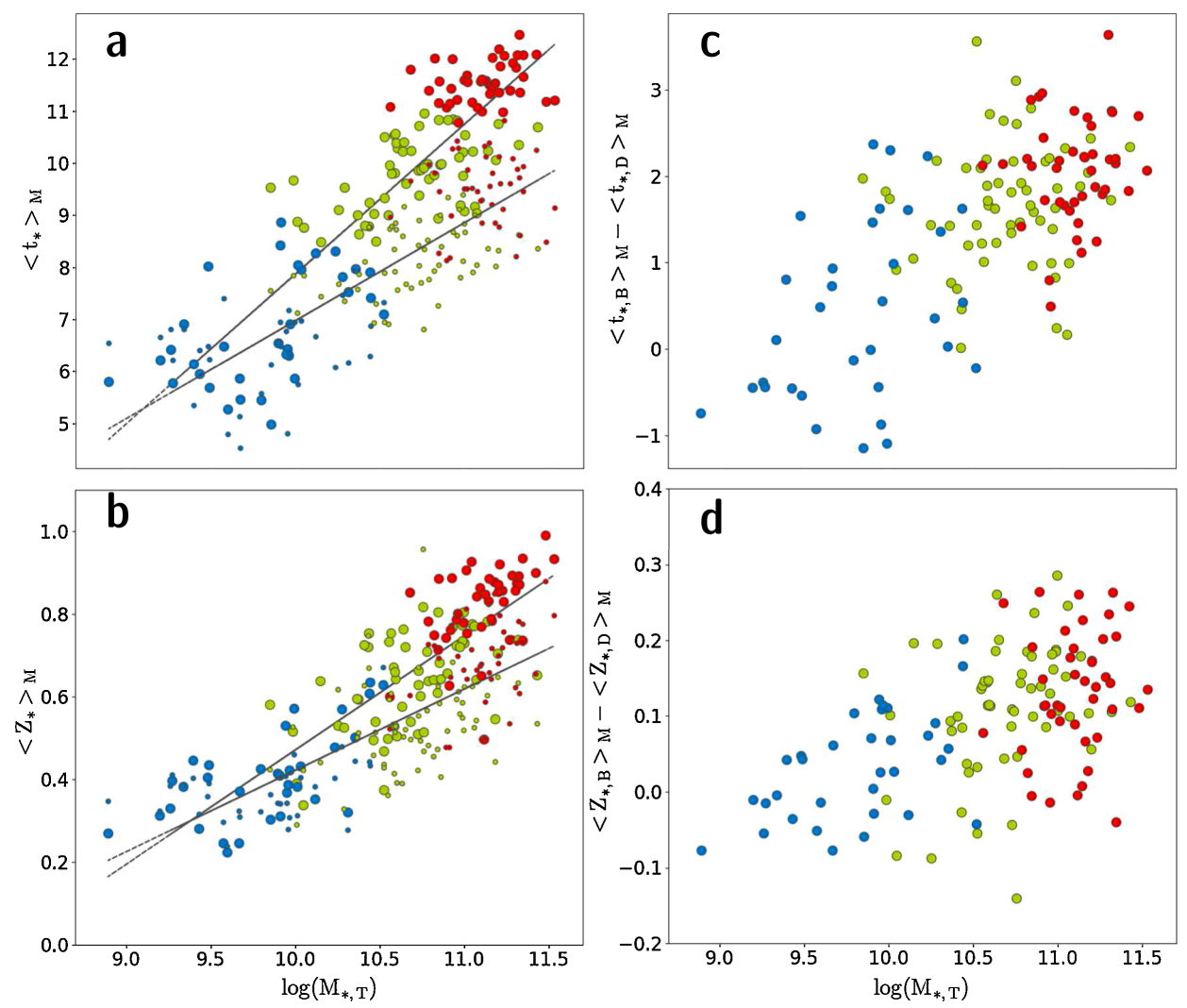

Fig. 7. Panel $a$ : logarithm of the total stellar mass $\mathcal{M}_{\star, T}\left(M_{\odot}\right)$ vs. mass-weighted stellar age $\left\langle t_{\star}\right\rangle_{\mathcal{M}}(\mathrm{Gyr})$ of the bulge and disk component (large and small dots, respectively). Linear fits to the data for the bulge and the disk are shown with solid lines. The color coding is the same as in Fig. 6. Panel b: $\log \left(\mathcal{M}_{\star, T}\right)$ vs. mass-weighted stellar metallicity $\left(Z_{\odot}\right)$ for the bulge and the disk. The layout is identical to that in panel a. Panel $c: \log \left(\mathcal{M}_{\star, \mathrm{T}}\right)$ vs. age contrast $\delta t_{\mathrm{BD}}\left(\left\langle t_{\star, \mathrm{B}}\right\rangle_{\mathcal{M}}\right.$ $\left.-\left\langle t_{\star, \mathrm{D}}\right\rangle_{\mathcal{M}}\right)(\mathrm{Gyr})$ between bulge and disk. Panel $d: \log \left(\mathcal{M}_{\star, T}\right)$ vs. metallicity contrast $\delta Z_{\mathrm{BD}}\left(\left\langle Z_{\star, \mathrm{B}}\right\rangle_{\mathcal{M}}-\left\langle Z_{\star, \mathrm{D}}\right\rangle_{\mathcal{M}}\right)$ in $Z_{\odot}$ between bulge and disk.

\section{Discussion}

In advance to the discussion below, it is worth recalling that this study combines, for the first time, three elements - surface photometry, spectral modeling of IFS data and post-processing of population vectors with $\mathcal{R} \mathcal{Y}$ - toward a systematic investigation of the evolutionary properties of LTG bulges within a uniformly defined isophotal radius that encompasses nearly their total emission.

\subsection{The continuous rise of bulges out of galactic disks}

The essential insight from Sect. 3 is that LTG bulges across $\sim 3$ dex in $\mathcal{M}_{\star}$ and $>1$ dex in $\Sigma_{\star}$ form a continuous sequence with regard to $\left\langle\delta \mu_{9 \mathrm{G}}\right\rangle,\left\langle t_{\star, \mathrm{B}}\right\rangle_{\mathcal{M}}$ and $\left\langle Z_{\star, \mathrm{B}}\right\rangle_{\mathcal{M}}$. This argues against an age bimodality that would echo two distinct bulge assembly routes, one directing to old, monolithically formed CBs and the other one to PBs emerging purely through quasi-continuous SF in the centers of secularly evolving disks. The combined evidence suggests instead that bulges and disks evolve alongside in a concurrent process that leads to a continuum of physical and evolutionary properties being reflected in $\left\langle\delta \mu_{9 \mathrm{G}}\right\rangle$ and exemplified by its three intervals (iA-iC) in Fig. 5. In high-mass $\left(\log \left(\mathcal{M}_{\star, \mathrm{B}}\right)>10\right)$ LTG bulges (interval iC), SF has shut off earlier than 9 Gyr ago, whereas in intermediate-mass ones (iB) it was prolonged to a later cosmic epoch, with the least massive bulges (iA) sustaining ongoing SF and being composed to $\sim 80 \%$ of stars younger than 9 Gyr. Additionally, the fact that older disks in our sample host older bulges (judging from a comparison of $m_{9}$ ratios and panels a and c of Fig. 7) with a within $\sim 0.2$ dex equal metallicity (panel d) points to an interwoven bulge-disk evolution, as already suggested by some previous photometric or spectroscopic studies in the local universe and at higher redshifts (e.g., Carollo et al. 2007; van Dokkum et al. 2013; Papovich et al.
2015; Sánchez-Blázquez et al. 2014; Sánchez-Blázquez 2016; Lang et al. 2014; Tacchella et al. 2018; Margalef-Bentabol et al. 2018, see also Sect. 1).

Our results are consistent with a picture where bulge growth in LTGs is driven by a superposition of quick-early and slowsecular processes, the relative importance of which increases with increasing galaxy mass. Quick-early processes might comprise collapse of self-gravitating gas that leaves behind a massive nuclear cluster or a low-mass proto-bulge, both providing a steep gravitational pool that could promote ensuing gas inflow and SF, eventually also the seed of a SMBH, in addition to inward migration and coalescence of massive $\left(\gtrsim 10^{8-9} M_{\odot}\right)$ SF clumps forming continuously out of violent disk instabilities (e.g., Bournaud et al. 2007; Mandelker et al. 2014, 2017, see Sect. 1). Inflowing inter-clump gas could add further fodder for the bulge buildup (e.g., Hopkins et al. 2012; Zolotov et al. 2015) during its dominant formation phase and beyond. As for slow-secular contributions to the bulge growth, these may comprise, according to our current understanding, steady gas inflow from the disk sustaining a perpetual rejuvenation of the bulge through in situ $\mathrm{SF}$, in concert with, for example, inward stellar migration and minor mergers of dwarf satellites (see Sect. 1). In terms of standard SFH parametrizations, the superposition of these processes might be empirically approximated by "delayed" exponentially declining SFR models (e.g., Cardoso et al. 2017) with an efolding timescale and a delay of the SFR peak scaling inversely to the present-day $\mathcal{M}_{\star, T}$. We note that the evidence from this study is qualitatively consistent with insights gained from Lick index studies by, for example, Thomas \& Davies (2006), which indicate that the smallest bulges show the lowest light-weighted ages and a late enrichment in iron from $\mathrm{SNe}$ Ia.

Clearly, a deeper understanding of the timescales and physical channels of bulge growth, including the possible regulatory role of AGN- and SF-driven feedback, is a fundamental yet 
formidable task that requires improved observational insights (for instance, on the mass function of SF clumps in high- $z$ protodisks; cf. e.g., Tamburello et al. 2017; Cava et al. 2017) and a detailed treatment of stellar-dynamical processes, such as stellar migration and its observational imprints (e.g., Sánchez-Blázquez et al. 2009; Roškar et al. 2012; Ruiz-Lara et al. 2016), the dissolution and regeneration of stellar bars (e.g., Berentzen et al. 2004), and, more generally, of all intrinsic and environmental parameters that shape the dynamical coupling of stars, gas and dark matter (DM) in an evolving galaxy ecosystem.

For example, a process that might act toward steepening the density profile of a bulge, thereby possibly promoting nuclear starburst activity (Loose et al. 1982), is adiabatic contraction of the stellar component in response to gas inflow. This effect, which was proposed by Papaderos et al. (1996b) as an explanation for the steeper stellar density profile of the underlying host of starbursting dwarfs, as compared to that of quiescent dwarf irregulars, might be relevant if accreted gas from the disk or directly from the cosmic web (e.g., Dekel et al. 2009; Dekel \& Burkert $2014)$ contributes a significant $(\gtrsim 30 \%)$ fraction of the dynamical mass in the central parts of proto-LTGs. This might be the case if baryons dominate in those radii (see Genzel et al. 2017, for recent observational evidence for baryon dominated disks 10 Gyr ago), or the DM halo has an extended core of nearly constant density (i.e., a Burkert 1995 profile, see also Salucci \& Burkert 2000).

\subsubsection{Star formation rate vs. stellar mass growth rate}

A subject of considerable interest is migration and its possible role on the mass growth rate (MGR) of LTG bulges. A subtle yet important point is that the MGR is not necessarily equal to the SFR, since inward (outward) migration across $R_{\mathrm{B}}$ may lead to a higher (lower) MGR than the value implied by in situ SF, after correction for the stellar mass fraction returned into the interstellar medium in the course of stellar evolution. The case of MGR $<$ SFR was discussed in Papaderos et al. (2002, see also Papaderos \& Östlin 2012) who conjectured that outward diffusion of newly formed stars in the starbursting cores of young dwarf galaxies may be an important driver of the buildup of their redder, more extended LSB stellar host: since the high-mass end of stellar populations with a lifetime shorter than a migration timescale $\tau_{\mathrm{m}}$ will be depopulated before reaching the LSB periphery, a stellar mass filtering effect will naturally lead to a radial (core-envelope) age (and color) gradient. A consequence of this is the overestimation of age for the LSB host, if its colors (or spectrum) are interpreted on the usual assumption of in situ SF according to a fully populated, spatially invariant initial mass function. The inverse situation (MGR $>$ SFR) might occur in the case of inward migration of ex situ formed SF clumps and stars from the disk, reaching the bulge with a delay $\tau_{\mathrm{m}}$ (presumably, greater than several $10^{8} \mathrm{yr}$ ) after their formation. Since this process imposes a minimum age $\tau_{\mathrm{m}}$ for stellar populations arriving at $R_{\mathrm{B}}$, inward migration into old, high- $\mathcal{M}_{\star, \mathrm{B}}$ and $-\Sigma_{\star, B}$ bulges could under certain circumstances act toward decreasing $\left\langle\delta \mu_{9 \mathrm{G}}\right\rangle, m_{9}$ and the bulge-to-disk age contrast $\delta t_{\mathrm{BD}}$. Following these considerations, the absence of stellar populations younger than $9 \mathrm{Gyr}$ in iC bulges does not necessarily rule out that their buildup has been continuing until $\left(9-\tau_{\mathrm{m}}\right)$ Gyr ago. Conversely, the presence of stars of age $\tau_{\mathrm{m}}$ in low-mass bulges does not imply that in situ SF in these entities has been ongoing already $\tau_{\mathrm{m}}$ Gyr ago. Summarizing, one effect that might be plausibly expected from inward migration is the aging (rejuvenation) of young (old) bulges by $\simeq \xi \cdot\left\langle\tau_{\mathrm{m}}\right\rangle$, whereby $\xi$ denotes the mass fraction of inwardly migrated stars or SF clumps, and $\left\langle\tau_{\mathrm{m}}\right\rangle$ is a mass-weighted average migration time for stars arriving in the bulge from different radii of the disk.

The sketchy remarks above are merely meant to outline one principle effect that inward migration might have on bulge age and SFH determinations, and to highlight the need for a better observational and theoretical understanding of this process. This task appears to be of considerable interest in the light of, for example, the recent conclusion by Margalef-Bentabol et al. (2018) that $\sim 1 / 2$ of the stellar mass formed in the disks of twocomponent galaxies at $1<z<3$ must have transferred inward, promoting the growth of the bulge.

\subsubsection{Accelerated bulge growth in proportion to galaxy mass}

From our previous remarks it follows that an enhanced MGR in the bulge could drive a non-homologous growth of radial $\Sigma_{\star}$ and $\mu$ profiles in LTGs: starting from an almost bulgeless exponential proto-disk with a Sérsic index $\eta \simeq 1$, an inwardly increasing MGR would act toward accelerating the buildup of the bulge and in turn lead to a steepening of the galaxy profile $(\eta>1)$ and gradual increase of the $\mathcal{M}_{\star, B} / \mathcal{M}_{\star, T}$ ratio. This is consistent with Fig. $6 \mathrm{c}$ that shows that high- $\left\langle\delta \mu_{9 \mathrm{G}}\right\rangle$ (iC) bulges hosted by massive $\left(\log \mathcal{M}_{\star} \simeq 11\right)$ LTGs contain twice the $\mathcal{M}_{\star, T}$ fraction than low- $\left\langle\delta \mu_{9 \mathrm{G}}\right\rangle$ (iA) bulges ( $\sim 40 \%$ and $\sim 20 \%$, respectively). The mass increase and compaction of the bulge to a high $\Sigma_{\star}$ might also support the central confinement and collapse of inflowing gas within its steepening gravitational potential, enhance chemical self-enrichment and therefore the gas cooling efficiency (cf., e.g., Böhringer \& Hensler 1989), which could in turn accelerate the SF cycle and the growth of $\mathcal{M}_{\star, B}$ in a manner that is proportional to the local $\Sigma_{\star}$. This process might continue until SF starts being gradually extinguished, once the bulge has attained a $\left(\log \mathcal{M}_{\star, \mathrm{B}} ; \log \Sigma_{\star, \mathrm{B}}\right) \geq(10 ; 9)$ and accretion-powered nuclear activity becomes important (cf. Sect. 4.2). The rise of bulges out of disks might therefore bear some analogy to (sub)galactic downsizing, in the sense that the assembly timescale of stellar systems scales inversely with the mass and initial density of the baryonic condensations galaxies emerge from, in qualitative resemblance to the staged galaxy formation scenario by Noeske et al. (2007).

Besides the trend for increasing $\left\langle\delta \mu_{9 \mathrm{G}}\right\rangle$ and $\left\langle t_{\star, \mathrm{B}}\right\rangle_{\mathcal{M}}$ with increasing $\mathcal{M}_{\star, \mathrm{B}}$ and $\mathcal{M}_{\star, \mathrm{T}}$ (Figs. 6a-c), further support to the notion of an accelerated bulge growth in proportion to the LTG mass comes from Fig. 7c that shows the relation between bulgeto-disk age contrast $\delta t_{\mathrm{BD}}$ as a function of $\mathcal{M}_{\star, \mathrm{T}}$ : would bulges form purely monolithically, prior to and independently of disks, then one would expect a roughly constant $(>0) \delta t_{\mathrm{BD}}$ across LTG mass. If, on the other hand, bulges were assembling strictly in lockstep with secularly evolving disks, thus sharing the same SFH and essentially being rearranged disk material, then their $\delta t_{\mathrm{BD}}$ would also be independent on $\mathcal{M}_{\star, \mathrm{T}}$ and in the range between $\approx 0$ Gyr and $\left\langle\tau_{\mathrm{m}}\right\rangle$. However, the documented trend for an increasing bulge-to-disk age contrast with increasing $\mathcal{M}_{\star, T}$ suggests that higher-mass bulges in higher-mass LTGs have assembled earlier and quicklier, and vice versa. We note that the relation between $\delta t_{\mathrm{BD}}$ and $\mathcal{M}_{\star, \mathrm{T}}$ (or, equivalently, $\left\langle\delta \mu_{9 \mathrm{G}}\right\rangle$; cf. Sect. 3) is unlikely to be driven by the earlier cessation of $\mathrm{SF}$ in higher-mass disks, since this would tend to diminish $\delta t_{\mathrm{BD}}$, contrary to the evidence from Fig. 7c. Quite importantly, the absence of a bimodality or even discontinuity in the bulge vs. disk age and metallicity contrast over $\sim 2.5 \operatorname{dex}$ in $\mathcal{M}_{\star, T}$ points to an interwoven yet asynchronous growth of these components, with bulges assembling out of disks on a timescale that scales 
inversely with $\mathcal{M}_{\star, T}$. In this sense, our results support neither the monolithic nor the purely secular bulge formation scenario, but point instead to a picture of sub-galactic downsizing where bulges form faster in more massive baryonic entities, and vice versa. The non-homologous radial growth of $\Sigma_{\star}$ implied by this bulge growth process is also consistent with the observed trend between the $\mathcal{M}_{\star, \mathrm{B}} / \mathcal{M}_{\star, \mathrm{T}}$ ratio and $\mathcal{M}_{\star, \mathrm{T}}$ (Fig. $6 \mathrm{c}$ ), as well as with a trend for an increasing Sérsic index $\eta$ with $\mathcal{M}_{\star, T}\left(\right.$ or $M_{r}$ ) for present-day LTGs.

The three LTGs in Fig. 5 may be regarded as morphological snapshots in a chronological sequence of increasing $\mathcal{M}_{\star, B}$ and $\Sigma_{\star, \mathrm{B}}$ in tandem with $\left\langle\delta \mu_{9 \mathrm{G}}\right\rangle$. Despite the tentative subdivision of LTG bulges into three intervals (iA-iC), for the sake of illustration, it is important to bear in mind that the here documented continuity in the bulge properties themselves and their difference $\left(\delta t_{\mathrm{BD}}\right.$ and $\left.\delta Z_{\mathrm{BD}}\right)$ to their hosting disks consistently disfavors a bulge segregation into evolutionary distinct classes, in particular between CBs and PBs. One may frame the insights from this study in a simple scenario where high-mass bulges $\left(\left\langle\delta \mu_{9 \mathrm{G}}\right\rangle\right.$ interval iC) represent the endpoint of an evolutionary pathway across an increasing $\left\langle\delta \mu_{9 \mathrm{G}}\right\rangle(\mathrm{iA} \rightarrow \mathrm{iB} \rightarrow \mathrm{iC})$ whose duration depends on the intrinsic and environmental properties of the halo in which LTGs form: higher-mass systems form preferentially in higher-density environments, where efficient gas accretion from the cosmic web (e.g., Dekel et al. 2009) facilitates rapid mass growth and complete the dominant phase of their bulge assembly earlier than 9 Gyr ago, while eventually maintaining a significant MGR in the ensuing Gyrs of their passive photometric evolution through inward stellar migration and minor dry mergers. Conversely, LTGs born out of lower-mass halos in less "privileged" regions of the cosmic web (filaments and their surroundings) might have experienced a delayed buildup as a result of comparatively inefficient gas accretion onto their shallow gravitational pools. The low "metabolism" (aka SSFR) of these latecomers in the iA interval of $\left\langle\delta \mu_{9 \mathrm{G}}\right\rangle$ is imprinted on their lower age, metallicity, stellar surface density and $\mathcal{M}_{\star, \mathrm{B}} / \mathcal{M}_{\star, \mathrm{T}}$ ratio. Moreover, the youth of these systems is not at odds with the presence of a minor fraction $(\sim 20 \%)$ of old (>9 Gyr) stars (cf. Sect. 3.3) that could originate from low-level SF and minor mergers with ancient galaxy building blocks, such as, for example, dwarf spheroidals formed prior to the reionization epoch (e.g., Grebel \& Gallagher 2004).

It is interesting to note that results from this study are qualitatively in line with conclusions drawn from abundance matching studies of higher- $z$ galaxies (e.g., van Dokkum et al. 2013; Patel et al. 2013; Papovich et al. 2015). For instance, Papovich et al. (2015) have investigated how the progenitors of present-day LTGs with the mass of the MW and M31 $\left(5 \times 10^{10} M_{\odot}\right.$ and $10^{11} M_{\odot}$, respectively) may have grown from $z=3$ to 0.5 . They report that both started their evolution as star-forming disks, and after an IR-luminous phase, they evolved in the redder and more quiescent galaxies of today. These authors further conclude that "the progenitors of MW-mass galaxies reached each evolutionary stage at later times (lower redshifts) and with stellar masses that are a factor of two to three lower than the progenitors of the M31-mass galaxies". Furthermore, these studies report a smooth increase of Sérsic $\eta$ with $z$, with higher-mass galaxies reaching a higher $\eta$ earlier than lower-mass galaxies, in agreement with the hypothesis of an accelerated bulge growth in proportion to galaxy mass suggested above.

\subsection{Accretion-powered nuclear activity along the bulge sequence}

The occurrence of accretion-powered nuclear activity in galactic bulges has been the subject of intense investigation over the

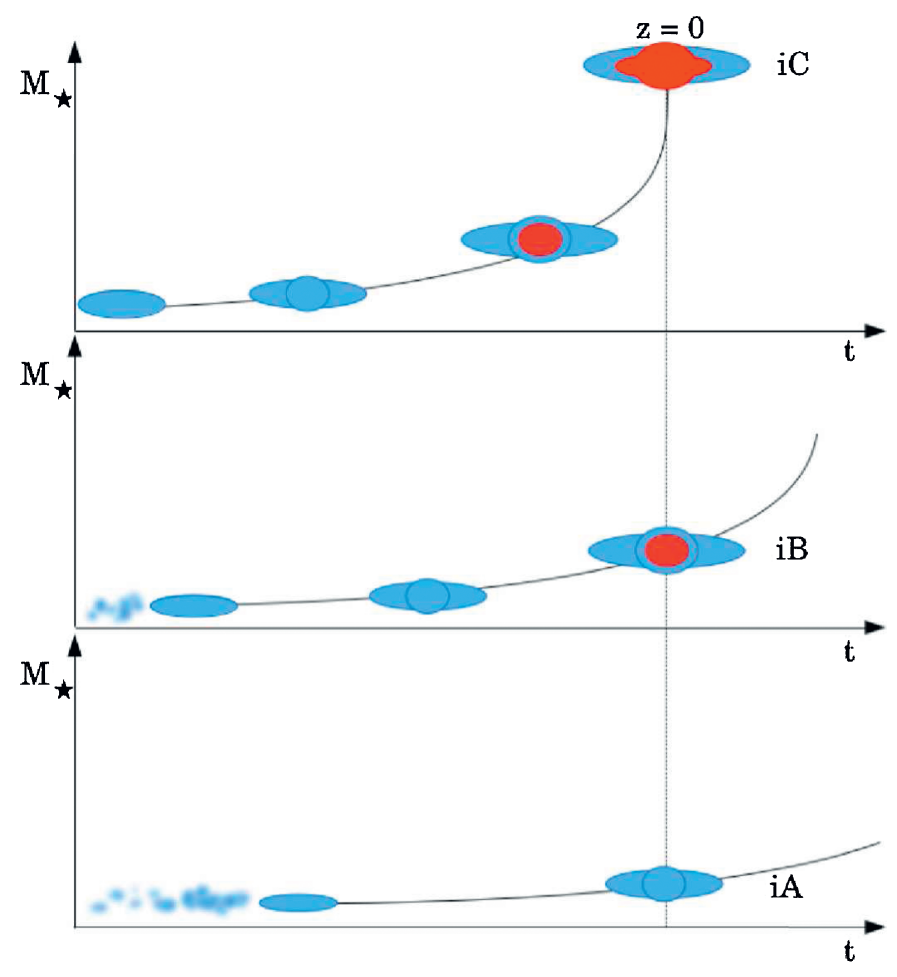

Fig. 8. Schematic illustration of the scenario proposed in Sect. 4.1.2: the three LTGs in Fig. 5 may be regarded as morphological snapshots in a sequence of increasing bulge stellar mass $\mathcal{M}_{\star, \mathrm{B}}$, age $\left\langle t_{\star, \mathrm{B}}\right\rangle_{\mathcal{M}}$, surface density $\Sigma_{\star, \mathrm{B}}$ and $\left\langle\delta \mu_{9 \mathrm{G}}\right\rangle$. The progenitors of high-mass LTGs (e.g., NGC 0776, i.e., systems in the $\left\langle\delta \mu_{9 \mathrm{G}}\right\rangle$ interval iC; $\geq-0.5 \mathrm{mag}$ ) form in more massive halos and experience the dominant phase of their bulge assembly earlier than $9 \mathrm{Gyr}$ ago (vertical line). Conversely, the precursors of low- $\left\langle\delta \mu_{9 \mathrm{G}}\right\rangle(\leq-1.5 \mathrm{mag}$; i.e., iA) LTGs, such as IC 0776 , assemble at a calm pace within lower-mass halos and undergo an overall retarded structural and chemical evolution toward intermediate$\left\langle\delta \mu_{9 \mathrm{G}}\right\rangle$ LTGs (iB), such as NGC 0001. Once the bulge has grown to $\left(\log \mathcal{M}_{\star, \mathrm{B}} ; \log \Sigma_{\star, \mathrm{B}}\right) \geq(10 ; 9), \mathrm{AGN}$-driven feedback initiates a gradual cessation of SF (depicted in red), while the bulge mass may continue increasing through inward stellar migration from the disk and multiple minor dry mergers.

past decades (the reader is referred to Kormendy \& Ho 2013, for a recent review) showing that manifestations of weak Seyfert activity are comparatively rare in disk-dominated LTGs that are commonly classified as PBs. Given the kinematical evidence for these bulges also containing a $\mathrm{SMBH}$, just like $\mathrm{CBs}$, the observed scarcity of AGN activity has been generally ascribed to an inefficient (sub-Eddington) and episodic matter accretion (e.g., Kormendy et al. 2011) or to the premature termination of SMBH growth, possibly due to the dissolution of short inner bars (Du et al. 2017).

This picture is echoed in Fig. 6f, showing that 31 of the 33 lower-mass iA bulges in our sample fall in the SF locus of the BPT diagram, with only two of them being spectroscopically classified as Composite. Assuming that the $M_{\bullet} / \mathcal{M}_{\star, T}$ ratio of $\approx 10^{-3}$ for galaxies with $\log \left(\mathcal{M}_{\star, T}\right) \geq 10$ (Kormendy \& Ho 2013) applies to lower masses, the $M_{\bullet}$ of $\sim 10^{6-7} M_{\odot}$ expected for iA bulges in our sample would translate to maximum Eddington rates of $0.02-0.2 M_{\odot} \mathrm{yr}^{-1}$, assuming a radiative efficiency of 0.1. Even though weak and smeared by the PSF of CALIFA IFS data, an AGN point source powered by these accretion rates should probably be detectable above the local background of these least massive bulges, given that their surface brightness is by 2-3 SDSS $r$ mag fainter than that of high-mass 
(iC) bulges. Moreover, the omnipresence of SF in these bulges, implying a cold gas reservoir of sufficient density, suggests that the absence of Seyfert signatures is not due to a high $\mathrm{Ly}_{c}$ continuum photon escape fraction (contrary to the case of many massive ETGs) or their dilution by circumnuclear SF (cf. discussion in Sect. 2.3). Moreover, an obscured AGN does neither offer a convincing explanation, given the relatively strong nebular emission $(\mathrm{EW}(\mathrm{H} \alpha)=26.3 \AA$, on average $)$, moderate-to-low $\left(A_{V} \leq 0.24 \pm 0.12 \mathrm{mag}\right)$ intrinsic extinction and nearly face-on geometry of these low- $\mathcal{M}_{\star, \mathrm{B}}$ LTGs. The evidence from Fig. 6f is certainly compatible with the picture of inefficient or sporadic gas accretion onto an intermediate- $M . \mathrm{SMBH}$, as suggested from previous work. A conceivable alternative, on the other hand, might be an asynchronous growth of SMBHs relative to their galaxy hosts with the $M_{\bullet} / \mathcal{M}_{\star, \mathrm{B}}$ ratio, or the Eddington ratio and the SMBH spin parameter being non-linearly coupled to $\mathcal{M}_{\star, T}$.

Coming to massive LTG bulges with a $\left\langle\delta \mu_{9 \mathrm{G}}\right\rangle$ above $-1.5 \mathrm{mag}$ ( $\mathrm{iB}$ and $\mathrm{iC}$ ), we recall that the gray-shaded quadrant in Fig. 6e contains 93\% (38/41) of all Seyfert and LINER in our sample. This suggests that the deep gravitational potential of bulges in the range $\log \left(\mathcal{M}_{\star, \mathrm{B}} ; \Sigma_{\star, \mathrm{B}}\right) \geq(10 ; 9)$ is linked, perhaps causally, to an efficient SMBH feeding. The regulatory role of the associated AGN phenomenon on SF quenching in these old, high-mass bulges remains a subject of intense investigation (e.g., Cattaneo et al. 2009; Kormendy \& Ho 2013; Harrison 2017, and references therein) both in the local universe and at intermediate $z$. For instance, Lang et al. (2014) study 6764 galaxies with $\mathcal{M}_{\star, \mathrm{T}}>10^{10} M_{\odot}$ at $0.5<z<2.5$ by performing bulge-to-disk decomposition. Their findings suggest that bulges and SMBHs grow hand in hand through merging and/or disk instabilities, with AGN-feedback being the main driver for the shut-off of SF. Tacchella et al. (2018) conjecture that SF quenching mechanisms must be internal to the galaxies and closely associated with bulge growth, and that, at $\mathcal{M}_{\star, \mathrm{T}}>10^{11} M_{\odot}$, SF quenching gradually progresses in an inside-out manner. This conclusion appears to be consistent with our results: 16 of the 76 LTGs in the gray-shaded area of Fig. 6e are classified as Composites, which points to the co-existence of SF with AGN-driven feedback over a time span of $\gtrsim 2$ Gyr (cf. panel $b$ with panel f), therefore against an abrupt SF quenching by accretion-powered nuclear activity.

\section{Summary and conclusions}

This study combines for the first time three techniques - surface photometry of SDSS data, 2D analysis and spectral modeling of IFS data and post-processing of the spectral synthesis output with the code $\mathcal{R} \mathcal{Y}$ - toward a systematic investigation of the physical and evolutionary properties (e.g., $\mathcal{M}_{\star}, t_{\star}, \Sigma_{\star}, Z_{\star}, M_{r}$, $R_{\mathrm{B}}$ ) of galaxy bulges within a uniformly defined isophotal radius that encompasses almost their total emission. Our sample is composed of 135 non-interacting, nearly face-on LTGs from the CALIFA survey that densely cover a wide range in bulge stellar mass $\left(10^{8.3}-10^{11.3} M_{\odot}\right)$ and luminosity $\left(-20 \lesssim M_{r} \lesssim-14\right)$.

A central element of our analysis revolves around $\left\langle\delta \mu_{9 \mathrm{G}}\right\rangle$ (mag), a quantity that can be inferred from $\mathcal{R} \mathcal{Y}$ and which allows to evaluate the contribution of stellar populations older than 9 Gyr to the mean $r$-band surface brightness of galaxy bulges. We show that this distance- and nominally extinctionindependent quantity, first introduced and systematically investigated for a large LTG sample here, offers a simple yet valuable semi-empirical proxy to the bulge assembly history, and eventually a promising bulge classification diagnostic.
The main results of this study may be summarized as follows: i. LTG bulges span a range in $\left\langle\delta \mu_{9 \mathrm{G}}\right\rangle$ between $\sim-4 \mathrm{mag}$ and $\sim 0$ mag, translating, respectively, into a contribution to the bulge mean surface brightness between $\sim 3 \%$ and $\sim 100 \%$ by stellar populations older than 9 Gyr. Quite importantly, $\left\langle\delta \mu_{9 \mathrm{G}}\right\rangle$ follows a tight correlation with the present-day stellar mass $\mathcal{M}_{\star, \mathrm{B}}$, stellar surface density $\Sigma_{\star, \mathrm{B}}$, mass-weighted age $\left\langle t_{\star, \mathrm{B}}\right\rangle_{\mathcal{M}}$ and metallicity $\left\langle Z_{\star, \mathrm{B}}\right\rangle_{\mathcal{M}}$ of LTG bulges over $\sim 3 \mathrm{dex}$ in $\mathcal{M}_{\star, \mathrm{B}}$ and $>1$ dex in $\Sigma_{\star, \mathrm{B}}$ : The highest- $\left\langle\delta \mu_{9 \mathrm{G}}\right\rangle(\sim 0 \mathrm{mag})$ bulges are the oldest, most massive, dense and chemically enriched, and vice versa.

ii. On the basis of $\left\langle\delta \mu_{9 \mathrm{G}}\right\rangle$, we tentatively subdivide LTG bulges in three characteristic intervals: low-mass $\left(\log \mathcal{M}_{\star, B} \lesssim 9.7\right)$ bulges with a $\left\langle\delta \mu_{9 \mathrm{G}}\right\rangle \leq-1.5 \mathrm{mag}$ (interval iA) are hosted by low-mass $\left(\log \mathcal{M}_{\star, T} \lesssim 10.5\right)$ disk-dominated LTGs, are comparatively young $\left(\left\langle t_{\star, B}\right\rangle_{\mathcal{M}} \simeq 6.8 \mathrm{Gyr}\right)$ and show ongoing SF with an average $\mathrm{EW}(\mathrm{H} \alpha) \simeq 25 \AA$. Conversely, highmass bulges $\left(\left\langle\delta \mu_{9 \mathrm{G}}\right\rangle \geq-0.5 \mathrm{mag}\right.$; interval iC) reside in highmass $\left(\log \mathcal{M}_{\star} \gtrsim 11\right)$ LTGs, have a mean age of $\simeq 11.5 \mathrm{Gyr}$ and are characterized by a high stellar mass surface density $\left(\Sigma_{\star, \mathrm{B}} \geq 10^{9} \quad M_{\odot} \mathrm{kpc}^{-2}\right)$ and weak nebular emission $(\langle\mathrm{EW}(\mathrm{H} \alpha)\rangle \simeq 5 \AA$. As for bulges in the intermediate range of $\left\langle\delta \mu_{9 \mathrm{G}}\right\rangle$ (interval iB), they also display intermediate values in $\mathcal{M}_{\star, \mathrm{B}}, \Sigma_{\star, \mathrm{B}},\left\langle t_{\star, \mathrm{B}}\right\rangle_{\mathcal{M}},\left\langle Z_{\star, \mathrm{D}}\right\rangle_{\mathcal{M}}$, absolute $r$-band magnitude and $\operatorname{EW}(\mathrm{H} \alpha)$.

iii. Whereas more massive bulges are hosted by more massive LTGs, the bulge-to-total mass ratio $\mathcal{M}_{\star, \mathrm{B}} / \mathcal{M}_{\star, \mathrm{T}}$ increases with LTG mass $\mathcal{M}_{\star, T}$, being on average twice as large in high- $\left\langle\delta \mu_{9 \mathrm{G}}\right\rangle \mathrm{iC}$ bulges than in low- $\left\langle\delta \mu_{9 \mathrm{G}}\right\rangle \mathrm{iA}$ bulges $(\sim 0.4$ and $\sim 0.2$, respectively).

iv. The age and metallicity of LTG bulges is moderately correlated with the age and metallicity of their parent disks. However, the bulge-to-disk age and metallicity contrast increases with $\mathcal{M}_{\star, T}$ and $\left\langle\delta \mu_{9 \mathrm{G}}\right\rangle$ : whereas the bulge and the disk are of similar age and metallicity in low-mass LTGs, high-mass bulges are, on average, by $\sim 2$ Gyr older and by 0.2 dex more metal-rich than the disk.

vi. An analysis of BPT diagnostics indicates that SF is the dominant gas excitation mechanism in lower-mass bulges. Accretion-powered nuclear activity, manifesting itself in Seyfert- and eventually LINER-specific BPT line ratios is almost exclusively confined to higher-mass $\left(\gtrsim 10^{10} M_{\odot}\right)$, higher- $\Sigma_{\star, \mathrm{B}}\left(\gtrsim 10^{9} M_{\odot} \mathrm{kpc}^{-2}\right)$ bulges.

The essential insight from this study is that LTG bulges across $\sim 3$ dex in $\mathcal{M}_{\star}$ and $>1$ dex in $\Sigma_{\star}$ form a continuous sequence with regard to $\left\langle\delta \mu_{9 \mathrm{G}}\right\rangle,\left\langle t_{\star, \mathrm{B}}\right\rangle_{\mathcal{M}}$ and $\left\langle Z_{\star, \mathrm{B}}\right\rangle_{\mathcal{M}}$. This argues against an age bimodality that would reflect two distinct bulge assembly routes, one directing to old, monolithically formed $\mathrm{CBs}$ and the other one to PBs emerging purely through quasi-continuous SF in the centers of secularly evolving disks. The combined evidence suggests instead that bulges and disks evolve alongside in a concurrent process that leads to a continuum of physical and evolutionary properties being closely reflected in $\left\langle\delta \mu_{9 \mathrm{G}}\right\rangle$. Our results are consistent with a picture where bulge growth in LTGs is driven by a superposition of quick-early and slow-secular processes, the relative importance of which is increasing with increasing galaxy mass.

Furthermore, the trend for an increasing $\mathcal{M}_{\star, \mathrm{B}} / \mathcal{M}_{\star, \mathrm{T}}$ ratio and bulge-to-disk age contrast with increasing $\mathcal{M}_{\star, T}$ (or, equivalently, $\left.\left\langle\delta \mu_{9 \mathrm{G}}\right\rangle\right)$, and the absence of a bimodality or even discontinuity in this relation over 3 dex in bulge mass, lend support to the interpretation of an interwoven yet asynchronous growth of bulge and disk, with the former assembling out of the latter 
I. Breda and P. Papaderos: The continuous rise of bulges out of galactic disks

on a timescale that is inversely related to $\mathcal{M}_{\star, T}$. This process is expected to lead to a non-homologous radial growth of $\Sigma_{\star}$ in LTGs and a trend for an increasing Sérsic index $\eta$ with increasing $\mathcal{M}_{\star, \mathrm{T}}$.

This framework points against a fundamental evolutionary dichotomy between CBs and PBs, instead unifying LTG bulges into a continuous chronological sequence of increasing $\mathcal{M}_{\star, B}$ and $\Sigma_{\star, \mathrm{B}}$ and inviting to a further exploration of the mechanisms behind the non-homologous growth of stellar mass in galaxies and the rise of bulges out of galactic disks.

Acknowledgements. We thank the anonymous referee for valuable suggestions and comments. This work was supported by Fundação para a Ciência e a Tecnologia (FCT) through national funds and by FEDER through COMPETE by the grants UID/FIS/04434/2013 \& POCI-01-0145-FEDER 007672 and PTDC/FIS-AST/3214/2012 \& FCOMP-01-0124-FEDER-029170. We acknowledge supported by European Community Programme ([FP7/20072013]) under grant agreement No. PIRSES-GA-2013-612701 (SELGIFS). IB was supported by the fellowship PD/BD/52707/2014 funded by FCT (Portugal) and POPH/FSE (EC) and by the fellowship CAUP-07/2014-BI in the context of the FCT project PTDC/FIS-AST/3214/2012 \& FCOMP-01-0124 FEDER-029170. PP was supported by FCT through Investigador FCT contract IF/01220/2013/CP1191/CT0002. Linear fits were computed with the code OK which was developed by Dr. Hans-Hermann Loose († August 5th, 1993). We thank Dr. Jean Michel Gomes for useful comments. This study uses data provided by the Calar Alto Legacy Integral Field Area (CALIFA) survey (http://califa.caha.es), funded by the Spanish Ministry of Science under grant ICTS-2009-10, and the Centro Astronómico Hispano-Alemán. It is based on observations collected at the Centro Astronómico Hispano Alemán (CAHA) at Calar Alto, operated jointly by the Max-Planck-Institut für Astronomie and the Instituto de Astrofísica de Andalucí a (CSIC). This research has made use of the NASA/IPAC Extragalactic Database (NED) which is operated by the Jet Propulsion Laboratory, California Institute of Technology, under contract with the National Aeronautics and Space Administration.

\section{References}

Aguerri, J. A. L., Balcells, M., \& Peletier, R. F. 2001, A\&A, 367, 428 Allen, M. G., Groves, B. A., Dopita, M. A., Sutherland, R. S., \& Kewley, L. J. 2008 ApJS, 178, 20

Asari, N. V., Cid Fernandes, R., Stasińska, G., et al. 2007, MNRAS, 381, 263

Baldwin, J. A., Phillips, M. M., \& Terlevich, R. 1981, PASP, 93, 5 (BPT)

Bell, E., Monachesi, A., Harmsen, B., et al. 2017, ApJ, 837, L1

Bender, R., Burstein, D., \& Faber, S. M. 1992, ApJ, 399, 462

Berentzen, I., Athanassoula, E., Heller, C. H., \& Fricke, K. J. 2004 MNRAS, 347,220

Binette, L., Magris, C. G., Stasińska, G., \& Bruzual, A. G. 1994, A\&A, 292, 13

Bird, J. C., Kazantzidis, S., \& Weinberg, D. H. 2012, MNRAS, 420, 913

Bournaud, F., Elmegreen, B. G., \& Elmegreen, D. M. 2007, ApJ, 670, 237

Böhringer, H., \& Hensler, G. 1989, A\&A, 215, 147

Breda, I. 2014, MSc Thesis, University of Porto

Brinchmann, J., Charlot, S., White, S. D. M., et al. 2004, MNRAS, 351, 1151

Bruzual, G., \& Charlot, S. 2003, MNRAS, 344, 1000

Burkert, A. 1995, ApJ, 447, L25

Cano-Díaz, M., Sánchez, S. S., Zibetti, S., et al. 2016, ApJ, 821, 26

Cardoso, L., Gomes, J. M., \& Papaderos, P. 2017, A\&A, 604, A99

Carollo, C. M., Stiavelli, M., de Zeeuw, P. T., Seigar, M., \& Dejonghe, H. 2001, ApJ, 546, 216

Carollo, C. M., Scarlata, C., Stiavelli, M., Wyse, R. F. G., \& Mayer, L. 2007, ApJ, 658, 960

Cattaneo, A., Faber, S. M., Binney, J., et al. 2009, Nature, 460, 213

Cava, A., Schaerer, D., Richard, J., et al. 2017, Nat. Astron., 2, 76

Cid Fernandes, R., Mateus, A., Sodré, L., Stasińska, G., \& Gomes, J. M. 2005, MNRAS, 358, 363

Cid Fernandes, R., Stasinka, G., Schlickmann, M. S., et al. 2010, MNRAS, 403, 1036

Cid Fernandes, R., Stasińska, G., Mateus, A., et al. 2011, MNRAS, 413, 1687

Cid Fernandes, R. González Delgado, R. García Benito, R., et al. 2014, A\&A, 561, A 130

Courteau, S., de Jong, R. S. , \& Broeils, A. H. 1996, ApJ, 457, L73

Dekel, A., \& Burkert, A. 2014 MNRAS, 438, 1870

Dekel, A., Birnboim, Y., Engel, G., et al. 2009, Nature, 457, 451

Dopita, M. A., \& Sutherland, R. S. 1995, ApJ, 455, 468
Drory, N., \& Fisher, D. 2007, ApJ, 664, 640

Du, M., Debattista, V. P., Shen, J., \& Cappellari, M. 2017, ApJ, 844, L15

Elmegreen, B. G., Bournaud, F., \& Elmegreen, D. M. 2008, ApJ, 688, 67 Erwin, P., 2015, ApJ, 799, 226

Fernandez Lorenzo, M., Sulentic, J., Verdes-Montenegro, L., et al. 2014, ApJ, 788, L39

Ferrarese, L., \& Merritt, D. 2000, ApJ, 539, L9

Fisher, D., \& Drory, N. 2010, ApJ, 716, 942

Fisher, D., \& Drory, N. 2011, ApJ, 733, L47

Förster Schreiber, N., Shapley, A. E., Genzel, R., et al. 2011, ApJ, 739, 45

Gadotti, D. A. 2009, MNRAS, 393, 1531

Gadotti, D. A. 2012, in Galaxy Bulges and Elliptical Galaxies - Lecture Notes, eds. G. Bono, \& M. Zoccali, [arXiv: 1208.2295]

Gadotti, D. A., \& dos Anjos, S. 2001, AJ, 122, 1298

Gallazzi, A., Charlot, S., Brinchmann, J., White, S. D. M., \& Tremonti, C. A. 2005, MNRAS, 362, 41

Carcía-Benito, R., Zibetti, S., Sánchez, S. F., et al. 2015, A\&A, 576, A135

Genzel, R, Förster Schreiber, N., Übler, H., et al. 2017, Nature, 543, 397

Gomes, J. M., \& Papaderos, P. 2016, A\&A, 594, A49

Gomes, J. M., Papaderos, P., Kehrig, C., et al. 2016a, A\&A, 588, A68

Gomes, J. M., Papaderos, P., Vílchez, C., et al. 2016b, A\&A, 586, A22

Grand, R. J. J., Daisuke, K., \& Cropper, M. 2014, MNRAS, 439, 623

González-Delgado, R. M., Cid Fernandes, R., Garcá-Benito, R., et al. 2014, ApJ, 791, L16

González-Delgado, R. M., Cid Fernandes, R., Peréz, E., et al. 2016, A\&A, 590, A44

Grebel, E. K., \& Gallagher, J. S. 2004 ApJ, 610, L89

Guedes, J., Mayer, L., Carollo, M., \& Madau, P. 2013, ApJ, 772, 36

Halle, A., Di Matteo, P., Haywood, M., \& Combes, F. 2015, A\&A, 578, A58

Harrison, C. 2017, Nat. Astron., 1E, 165H

Heavens, A., Panter, B., Jimenez, R., \& Dunlop, J. 2004, Nature, 428, 625

Heckmann, T. M. 1980, A\&A, 87, 152

Ho, L. C. 1999, Adv. Space Res., 23, 813

Ho, L. C. 2008, ARA\&A, 46, 475

Hopkins, P. F, Kereš, D., Murray, N., Quataert, E., \& Hernquist, L. 2012, MNRAS, 427, 968

Iglésias-Páramo, J., Vílchez, J. M., Rosales-Ortega, F. F., et al. 2016, ApJ, 826, 71

Johnston, E. J., Aragón-Salamanca, A., Merrifield, M. R., \& Bedregal, A. G. 2012, MNRAS, 422, 2590

Johnston, E. J., Aragón-Salamanca, A., \& Merrifield, M. R. 2014, MNRAS, 441, 333

Johnston, E. J., Häußler, B., Aragón-Salamanca, A., et al. 2017, MNRAS, 465, 2317

Kauffmann, G., Heckman, T. M., Tremonti, C., et al. 2003, MNRAS, 346, 1055 Kauffmann, G., Heckman, T. M., White, S. D. M., et al. 2013, MNRAS, 341, 54 Kehrig, C., Monreal-Ibero, A., Papaderos, P., et al. 2012, A\&A, 540, A11 Kelz, A., Verheijen, M. A. W., Roth, M. M., et al. 2006, PASP, 118, 129 Keselman, J. A., \& Nusser, A. 2012, MNRAS, 424, 1232

Kewley, L. J., Dopita, M. A., Sutherland, R. S., Heisler, C. A., \& Trevena, J. 2001, ApJ, 556, 121

Koleva, M., Prugniel, P., Ocvirk, P., et al. 2008, MNRAS, 385, 1998

Kormendy, J. 1977, ApJ, 217, 406

Kormendy, J., \& Kennicutt, Jr., R. 2004, ARA\&A, 42, 603

Kormendy, J., \& Ho, L. C. 2013, ARA\&A, 51, 511

Kormendy, J., Bender, R., \& Cornell, M. 2011, Nature, 469, 374

Kotilainen, J. K., León-Tavares, J., Olguín-Iglesias, A., et al. 2016, ApJ, 832, 157

Lang, P., Wuyts, S., Somerville, R. S., et al. 2014, ApJ, 788, 11

Larson, R. 1974, MNRAS, 166, 585

Loose, H. H., Krügel, E., \& Tutukov, A. 1982, A\&A 105, 342

Macchetto, F., Pastoriza, M., Caon, N., et al. 1996, A\&AS, 120, 463

Mandelker, N., Dekel, A., Ceverino, D., et al. 2014, MNRAS, 443, 3675

Mandelker, N., Dekel, A, Ceverino, D., et al. 2017, MNRAS, 464, 635

Margalef-Bentabol, B., Conselice, C., Mortlock, A., et al. 2016, MNRAS, 461, 2728

Margalef-Bentabol, B., Conselice, C., Mortlock, A., et al. 2018, MNRAS, 473, 5370

Méndez-Abreu, J., Aguerri, J. A. L., Corsini, E. M., \& Simonneau, E. 2008, A\&A, 478, 353

Mishra, P., Wadadekar, Y., \& Barway, S. 2017, MNRAS, 467, 2384

Moorthy, B. K., \& Holtzman, J. A. 2006, MNRAS, 371, 583

Morelli, L., Corsini, E. M., Pizzella, A., et al. 2012, MNRAS, 423, 962

Morelli, L., Parmiggiani, M., Corsini, E. M., et al. 2016, MNRAS, 463, 4396

Neumann, J., Wisotzki, L., Cloudhury, O. S., et al. 2017, A\&A, 604, A30

Ness, M., Debattista, V. P., Bensby, T., et al. 2014, ApJ, 787, L19

Noeske, K. G., Papaderos, P., Cairós, L. M., \& Fricke, K. J. 2003, A\&A, 410, 481

Noeske, K. G., Faber, S. M., Weiner, B. J., et al. 2007, ApJ, 660, L47

Noguchi, M. 1999, ApJ, 514, 77 
Okamoto, T. 2013, MNRAS, 428, 718

Papaderos, P., \& Östlin, G. 2012, A\&A, 537, A126

Papaderos, P., Loose, H. H., Thuan, T. X., \& Fricke, K. J. 1996a, A\&AS, 120, 207

Papaderos, P., Loose, H. H., Fricke, K. J., \& Thuan, T. X. 1996b, A\&A 314, 59

Papaderos, P., Izotov, Y. I., Thuan, T. X., et al. 2002, A\&A, 393, 461

Papaderos, P., Guseva, N. G., Izotov, Y. I., et al. 2006, A\&A, 457, 45

Papaderos, P., Gomes, J. M., Vílchez, J., et al. 2013, A\&A, 555, L1

Papovich, C., Labbé, I., Quadri, R., et al. 2015, ApJ, 803, 26

Patel, S. G., van Dokkum, P. G., Franx, M., et al. 2013, ApJ, 766, 15

Peletier, R. F., \& Balcells, M. 1996, AJ, 111, 2238

Peletier, R. F., Falcón-Barroso, J., Bacon, R., et al. 2007, MNRAS, 379, 445

Peng, C. Y, Ho, L. C., Impey, C. D., \& Rix, H. W. 2010, AJ, 139, 2097

Petropoulou, V., Vílchez, J., Iglésias-Páramo, J., et al. 2011, ApJ, 734, 32

Ribeiro, B., Lobo, C., Antón, S., Gomes, J. M., \& Papaderos, P. 2016, MNRAS, 456, 3899

Roškar, R., Debattista, V. P., Quinn, T. R., \& Wadsley, J. 2012, MNRAS, 426, 2089

Roth, M. M., Kelz, A., Fechner, T., et al. 2005, PASP, 117, 620

Ruiz-Lara, T., Pérez, I., Florido, E., et al. 2016, MNRAS, 456, L35

Salucci, P., \& Burkert, A. 2000, ApJ, 537, L9

Sánchez, S. F., Kennicutt, R. C., Gil de Paz, A., et al. 2012, A\&A, 538, A8

Sánchez, S. F., García-Benito, R., Zibetti, S., et al. 2016, A\&A, 594, A36

Sánchez-Blázquez, P. 2016, in Galactic Bulges, Astrophysics and Space Science Library (Berlin: Springer), 418, 127

Sánchez-Blázquez, P., Courty, S., Gibson, B. K., \& Brook, C. B. 2009, MNRAS, 398,591
Sánchez-Blázquez, P., Rosales-Ortega, F. F., Méndez-Abreu, J., et al. 2014, A\&A, 570, A6

Sarzi, M., Shields, J. C., Schawinski, K., et al. 2010, MNRAS, 402, 2187

Scannapieco, C., Gadotti, D. A., Jonsson, P., \& White, S. D. M. 2010, MNRAS, 407, 41

Schawinski, K., Thomas, D., Sarzi, M., et al. 2007, MNRAS, 382, 1415

Sérsic, J. L. 1963, Boletin de la Asociacion Argentina de Astronomia, 6, 41

Shapiro, K. L., Falcón-Barroso, J., van de Ven, G., et al. 2010, MNRAS, 402, 2140

Springel, V., \& Hernquist, L. 2005, ApJ, 622, L9

Stasińska, G, Vale Asari, N., Cid Fernandes, R., et al. 2008, MNRAS, 391, L29

Tacchella, S., Carollo, C., \& Schreiber, N. 2018, ApJ, 859, 56

Tamburello, V., Rahmati, A., Mayer, L., et al. 2017, MNRAS, 468, 4792

Thomas, D., \& Davies, R. 2006, MNRAS, 366, 510

Tissera, P. B., Machado, R. E. G., Sánchez-Blazquez, P., et al. 2016, A\&A, 592, A93

Tremonti, C. A., Heckman, T. M., Kauffmann, G., et al. 2004, ApJ, 613, 898

Trinchieri, G., \& di Serego Alighieri, S. 1991, AJ, 101, 1647

van Dokkum, P. G., Leja, J., Nelson, E. J., et al. 2013, ApJ, 771, L35

Verheijen, M., Bershady, M., Andersen, D., et al. 2004, Astron. Nachr., 325, 151

Worthey, G., Faber, S. M., Gonzalez, J. J., \& Burstein, D. 1994, ApJS, 94, 687

Wyse, R. F. G., Gilmore, G., \& Franx, M. 1997, ARA\&A, 35, 637

Wuyts, S., Förster Schreiber, N., Genzel, R., et al. 2012, ApJ, 753, 114

Yan, R., \& Blanton, M. R. 2012, ApJ, 747, 61

Zhao, Y. 2012, Ap\&SS, 337, 719

Zibetti, S., Gallazzi, A. R., Ascasibar, Y., et al. 2017, MNRAS, 468, 1902

Zolotov, A., Dekel, A., Mandelker, N., et al. 2015, MNRAS, 450, 2327 

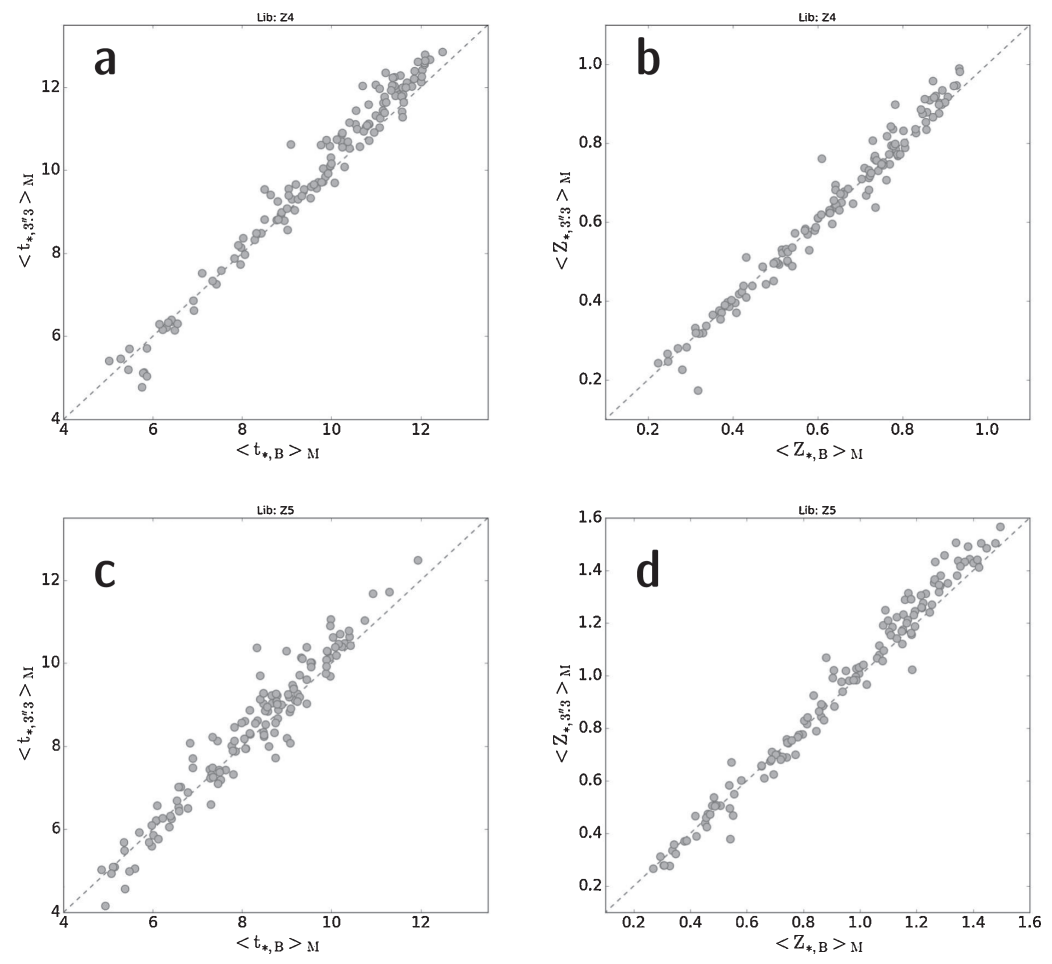

Fig. A.1. Comparison of the mean mass-weighted stellar age $\left\langle t_{\star, \mathrm{B}}\right\rangle_{\mathcal{M}}(\mathrm{Gyr})$ and metallicity $\left\langle Z_{\star, \mathrm{B}}\right\rangle_{\mathcal{M}}\left(Z_{\odot}\right)$ within $R_{\mathrm{B}}$ (cf. Sect. 2.2.2) with the values obtained within a radius of 3'.3 for the LTG bulges in our sample $\left(\left\langle t_{\star, 3.3}\right\rangle_{\mathcal{M}}\right.$ and $\left\langle Z_{\star, 3.3}\right\rangle_{\mathcal{M}}$, respectively). Dashed diagonal lines indicate equality between both plotted quantities for spectral models based on the Z4 and Z5 SSP library (upper and lower pan$e l s$, respectively). It can be seen that the scatter of points relative to the equality lines is relatively small (standard deviation of $0.56 \mathrm{Gyr}$ and 0.33 dex for spectral fits with the Z4 SSP library and $0.63 \mathrm{Gyr}$ and 0.60 dex for spectral fits with the Z5 SSP library) and does not show a significant dependence on age and metallicity.
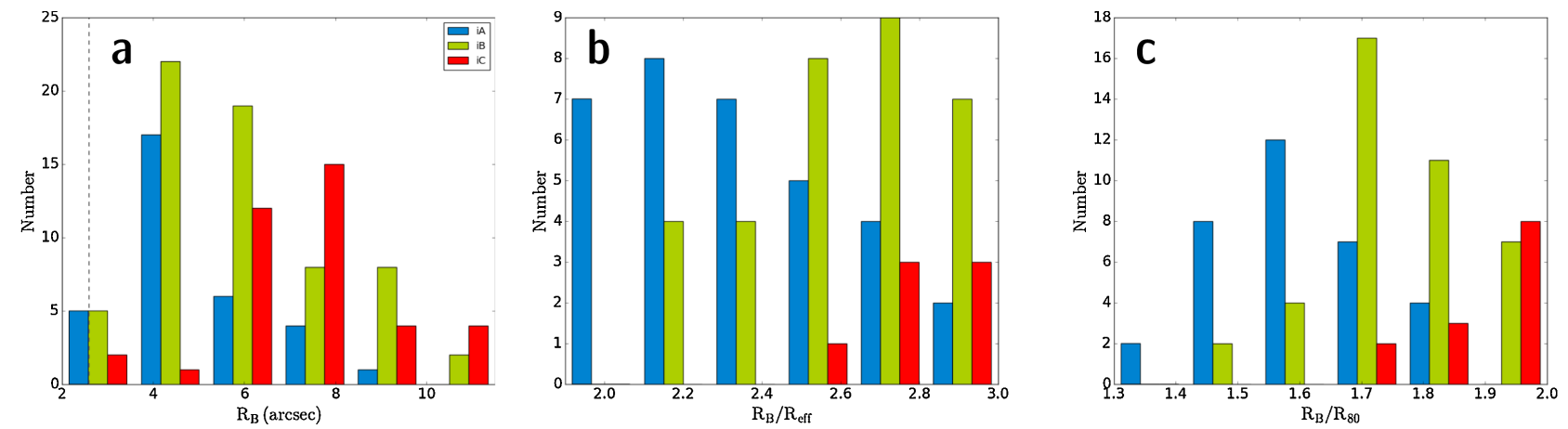

Fig. A.2. Histogram distributions of the bulge isophotal radius $R_{\mathrm{B}}\left({ }^{\prime \prime}\right)$ of the LTGs in our sample (panel a), and its normalized values to $R_{\mathrm{eff}}$ and the radius enclosing $80 \%$ of its total bulge luminosity (panels $b$ and $c$, respectively). The dashed vertical line in panel a marks the FWHM of CALIFA DR3 data (2".6). The color coding is identical to that in Fig. 6 and is meant to illustrate the variation of the bulge extent among the three tentatively defined $\left\langle\delta \mu_{9 \mathrm{G}}\right\rangle$ intervals (cf. Sect. 3).

\section{Appendix A: An empirical assessment of disk contamination and PSF convolution effects on the obtained trends}

Even though all but one $\left(\left\langle\delta \mu_{9 \mathrm{G}}\right\rangle\right)$ of the quantities involved in this study are weighted by $\mathcal{M}_{\star}$, therefore relatively insensitive to the luminosity contribution by the star-forming disk, it is worthwhile to check whether the latter has an appreciable impact on $\left\langle t_{\star, \mathrm{B}}\right\rangle_{\mathcal{M}}$ and $\left\langle Z_{\star, \mathrm{B}}\right\rangle_{\mathcal{M}}$ determinations within the bulge radius $R_{\mathrm{B}}$. In particular, since the $\mathrm{B} / \mathrm{T}$ mass ratio increases by a factor of $\sim 2$ across the range in $\mathcal{M}_{\star, T}$ covered by the analyzed LTG sample $\left(10^{9} M_{\odot}-10^{11.5} M_{\odot}\right)$, it cannot be excluded that the degree of disk contamination scales inversely with $\mathcal{M}_{\star, \mathrm{T}}$, therefore primarily affecting low-B/T (i.e., low- $\left\langle\delta \mu_{9 \mathrm{G}}\right\rangle \mathrm{iA}$ ) galaxies and leading to an artificial steepening of the $\log \left(\mathcal{M}_{\star, \mathrm{B}}\right)$ vs. $\left\langle t_{\star, \mathrm{B}}\right\rangle_{\mathcal{M}}$ relation in Fig. 6b.

For this purpose, a series of tests was made to study the dependence of $\left\langle t_{\star, \mathrm{B}}\right\rangle_{\mathcal{M}}$ and $\left\langle Z_{\star, \mathrm{B}}\right\rangle_{\mathcal{M}}$ on the isophotal aperture considered by repeating the analysis for the three innermost isan, as well as within the central portion of the bulge where contamination by the disk should be minimal. For the latter tests we adopted a radius of 3 .' 3 (hereafter $R_{3.3}$ ), which, given the typical full width at half maximum (FWHM) of CALIFA DR3 data $(\simeq 2$ '. 6$)$, encompasses the total emission of a point-like source (e.g., a compact bulge).

As apparent from Fig. A.1, mass-weighted age and metallicity determinations within $R_{3.3}$ do not appreciably differ from those within $R_{\mathrm{B}}$, which suggests that the trends in Figs. 6 and 7 are not notably affected by a $\mathcal{M}_{\star, T}$-dependent light contamination of the bulge by the surrounding star-forming disk.

The good agreement between $\left\langle t_{\star, \mathrm{B}}\right\rangle_{\mathcal{M}}$ and $\left\langle Z_{\star, \mathrm{B}}\right\rangle_{\mathcal{M}}$ determinations within $R_{3.3}$ and $R_{\mathrm{B}}$ is probably not due to point spread function (PSF) smearing effects, but presumably the result of weak radial gradients of mass-weighted quantities within LTG bulges. Indeed, the bulge radius in the majority $(\sim 95 \%)$ of the analyzed LTGs is by a factor 2-4.6 larger than the FWHM of CALIFA IFS data (vertical dashed line in panel a of Fig. A.2). Also, panels b\&c show that $R_{\mathrm{B}}$ is typically $\sim 2-3$ times larger 

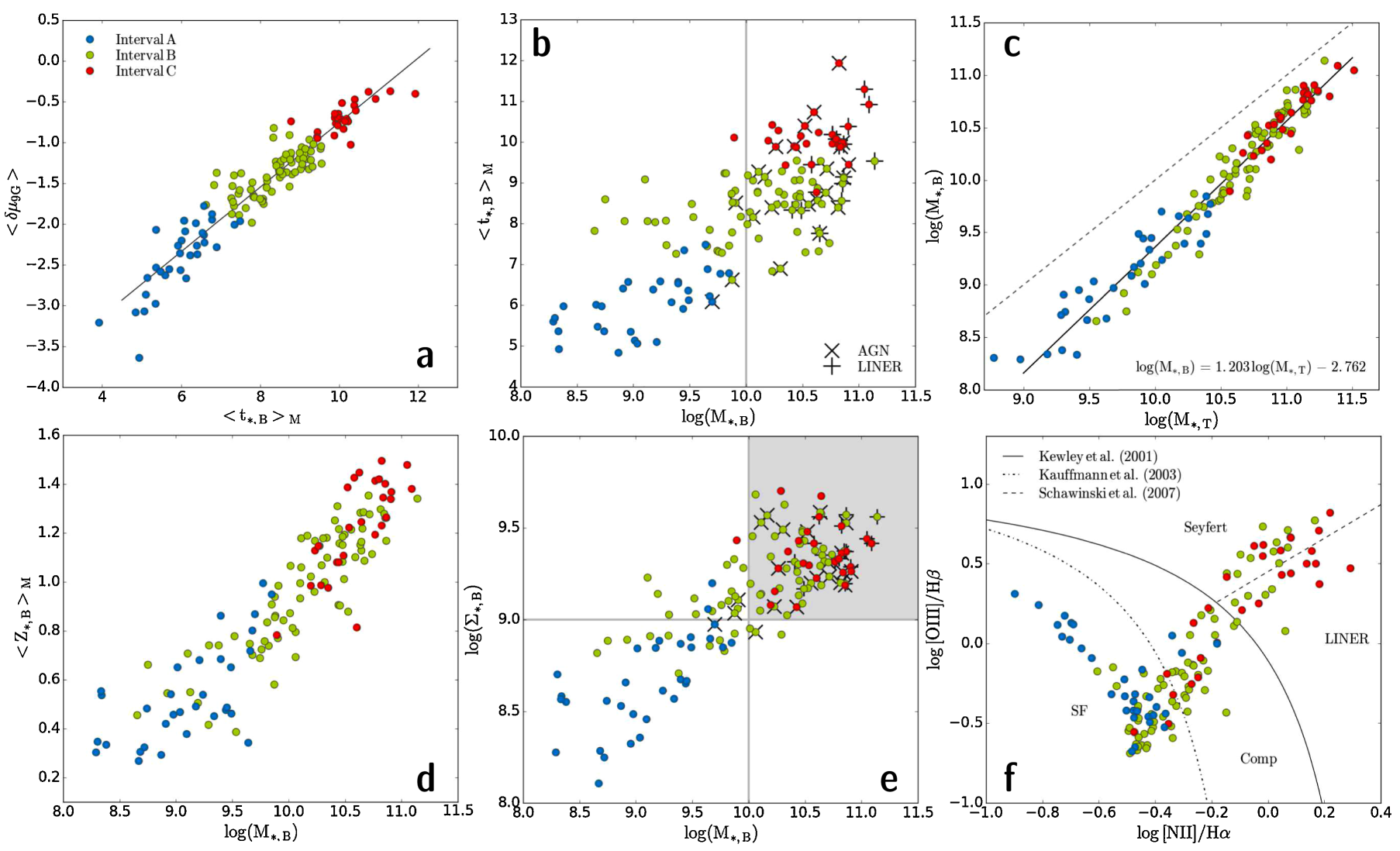

Fig. B.1. Main results from the spectral fitting of the LTG sample with the SSP base Z5 (cf. Sect. 2.2.2). The color coding and the meaning of the individual panels is as in Fig. 6. Panel $a$ : $\left\langle\delta \mu_{9 \mathrm{G}}\right\rangle$ ( $r$ mag) vs. mass-weighted stellar age $\left\langle t_{\star, \mathrm{B}}\right\rangle_{\mathcal{M}}$ (Gyr) for the bulge component, with the solid line showing a linear fit to the data. Panel $b$ : logarithm of the stellar mass $\mathcal{M}_{\star, \mathrm{B}}\left(M_{\odot}\right)$ in the bulge vs. mass-weighted stellar age $\left\langle t_{\star, \mathrm{B}}\right\rangle_{\mathcal{M}}(\mathrm{Gyr})$. Galaxies spectroscopically classified as Seyfert and LINER (cf. panel f) are marked. Panel $c$ : total stellar mass $\mathcal{M}_{\star, \mathrm{T}}$ vs. $\mathcal{M}_{\star, \mathrm{B}}$. The solid and dashed lines show, respectively, a linear fit to the data and equality between both quantities. Panel $d$ : Logarithm of $\mathcal{M}_{\star, \mathrm{B}}$ vs. mass-weighted stellar metallicity $\left\langle Z_{\star, \mathrm{B}}\right\rangle_{\mathcal{M}}\left(Z_{\odot}\right)$. Panel $e$ : logarithm of $\mathcal{M}_{\star, \mathrm{B}}$ vs. logarithm of the mean stellar surface density $\Sigma_{\star, \mathrm{B}}\left(M_{\odot} \mathrm{kpc}^{-2}\right)$ in the bulge. Panel $f$ : spectroscopic classification after BPT line ratios within simulated 3" SDSS fibers (cf. Sect. 2.3).

than the effective radius $R_{\mathrm{eff}}$ and contains in all cases at least $80 \%$ of the total emission of the bulge.

\section{Appendix B: Dependence of the inferred trends on the metallicity range covered by the SSP library}

As pointed out in Sect. 2.2.2, in our study preference was given to determinations that are based on spectral modeling with the Z4 SSP library with the goal of improving age determinations at the expense of a possible saturation of the metallicity at $\leq Z_{\odot}$. Nevertheless, a parallel spectral modeling analysis of the LTG sample was carried out using the Z5 SSP library, which additionally comprises SSPs for a stellar metallicity of $1.5 Z_{\odot}$, in order to evaluate the influence of the AMD on the trends discussed in Sect. 3.
As it can be seen from comparison of Fig. 6 with Fig. B.1, the AMD is reflected in an increase of the metallicity and decrease in the age of high- $\mathcal{M}_{\star}$ bulges. However, spectral modeling with the Z5 SSP library has a relatively little effect on $\left\langle\delta \mu_{9 \mathrm{G}}\right\rangle$ ( $\left.\pm 0.5 \mathrm{mag}\right)$ and $\mathcal{M}_{\star}(\$ 0.2-0.3 \mathrm{dex})$, and, quite importantly, it does not appreciably influence the trends between $\left\langle\delta \mu_{9 \mathrm{G}}\right\rangle$ and physical and evolutionary quantities of LTG bulges.

For the sake of completeness, we provide in Table B.1 a synopsis of relations inferred from regression analysis for quantities obtained from spectral modeling with the Z4 and Z5 SSP library (panels a-e in Figs. 3 and B.1, respectively). The upper block lists relations computed by minimizing $\chi^{2}$ with regard to both quantities considered, whereas the lower block refers to fits where $\chi^{2}$ minimization was done only with regard to the quantity listed in the right-hand side of each equation. 
Table B.1. Overview of the fits obtained for quantities inferred from analysis of the LTG sample with the Z4 and Z5 SSP library.

\begin{tabular}{|c|c|}
\hline brary & ry \\
\hline $\begin{array}{c}\left\langle t_{\star, \mathrm{B}}\right\rangle_{\mathcal{M}}=(2.05 \pm 0.04) \cdot\left\langle\delta \mu_{9 \mathrm{G}}\right\rangle+(12.14 \pm 0.06) \\
\left\langle t_{\star, \mathrm{B}}\right\rangle_{\mathcal{M}}=(2.05 \pm 0.04) \cdot\left\langle\delta \mu_{9 \mathrm{G}}\right\rangle+(12.14 \pm 0.06) \\
\left\langle t_{\star, \mathrm{B}}\right\rangle_{\mathcal{M}}=(2.30 \pm 0.11) \cdot \log \mathcal{M}_{\star, \mathrm{B}}-(13.44 \pm 1.13) \\
\log \Sigma_{\star, \mathrm{B}}=(0.42 \pm 0.02) \cdot \log \mathcal{M}_{\star, \mathrm{B}}+(4.96 \pm 0.23) \\
\log \Sigma_{\star, \mathrm{B}}=(0.16 \pm 0.01) \cdot\left\langle t_{\star, \mathrm{B}}\right\rangle_{\mathcal{M}}+(7.63 \pm 0.09) \\
\log \Sigma_{\star, \mathrm{B}}=(0.32 \pm 0.02) \cdot\left\langle\delta \mu_{9 \mathrm{G}}\right\rangle+(9.56 \pm 0.03) \\
\left\langle Z_{\star, \mathrm{B}}\right\rangle_{\mathcal{M}}=(0.23 \pm 0.01) \cdot \log \mathcal{M}_{\star, \mathrm{B}}-(1.60 \pm 0.11) \\
\left\langle Z_{\star, \mathrm{B}}\right\rangle_{\mathcal{M}}=(0.28 \pm 0.01) \cdot \log \mathcal{M}_{\star, \mathrm{T}}-(2.30 \pm 0.15) \\
\left\langle t_{\star, \mathrm{D}}\right\rangle_{\mathcal{M}}=(1.88 \pm 0.12) \cdot \log \mathcal{M}_{\star, \mathrm{T}}-(11.85 \pm 1.32) \\
\left\langle Z_{\star, \mathrm{D}}\right\rangle_{\mathcal{M}}=(0.20 \pm 0.02) \cdot \log \mathcal{M}_{\star, \mathrm{T}}-(1.55 \pm 0.16) \\
\log \mathcal{M}_{\star, \mathrm{B}}=(0.67 \pm 0.04) \cdot\left\langle\delta \mu_{9 \mathrm{G}}\right\rangle+(10.87 \pm 0.06) \\
\log \mathcal{M}_{\star}=(1.22 \pm 0.02) \log \mathcal{M}_{\star, \mathrm{T}}-(2.87 \pm 0.25) \\
\mathcal{M}_{\star, \mathrm{B}} / \mathcal{M}_{\star, \mathrm{T}}=(0.13 \pm 0.02) \cdot \log \mathcal{M}_{\star, \mathrm{T}}-(1.12 \pm 0.17) \\
\left\langle t_{\star, \mathrm{B}}\right\rangle_{\mathcal{M}}=(1.21 \pm 0.06) \cdot\left\langle t_{\star, \mathrm{D}}\right\rangle_{\mathcal{M}}-(0.18 \pm 0.49) \\
\left\langle Z_{\star, \mathrm{B}}\right\rangle_{\mathcal{M}}=(1.09 \pm 0.05) \cdot\left\langle Z_{\star, \mathrm{D}}\right\rangle_{\mathcal{M}}+(0.05 \pm 0.03) \\
\delta t_{\mathrm{BD}}=(1.00 \pm 0.12) \cdot \log \mathcal{M}_{\star, \mathrm{T}}-(9.07 \pm 1.28) \\
\delta Z_{\mathrm{BD}}=(0.08 \pm 0.01) \cdot \log \mathcal{M}_{\star, \mathrm{T}}-(0.75 \pm 0.12)\end{array}$ & $\begin{array}{c}\left\langle t_{\star, \mathrm{B}}\right\rangle_{\mathcal{M}}=(2.26 \pm 0.07) \cdot\left\langle\delta \mu_{9 \mathrm{G}}\right\rangle+(11.52 \pm 0.11) \\
\left\langle t_{\star, \mathrm{B}}\right\rangle_{\mathcal{M}}=(2.26 \pm 0.07) \cdot\left\langle\delta \mu_{9 \mathrm{G}}\right\rangle+(11.52 \pm 0.11) \\
\left\langle t_{\star, \mathrm{B}}\right\rangle_{\mathcal{M}}=(1.69 \pm 0.12) \cdot \log \mathcal{M}_{\star, \mathrm{B}}-(8.71 \pm 1.23) \\
\log \Sigma_{\star, \mathrm{B}}=(0.39 \pm 0.02) \cdot \log \mathcal{M}_{\star, \mathrm{B}}+(5.20 \pm 0.24) \\
\log \Sigma_{\star, \mathrm{B}}=(0.16 \pm 0.01) \cdot\left\langle t_{\star, \mathrm{B}}\right\rangle_{\mathcal{M}}+(7.76 \pm 0.10) \\
\log \Sigma_{\star, \mathrm{B}}=(0.39 \pm 0.03) \cdot\left\langle\delta \mu_{9 \mathrm{G}}\right\rangle+(9.69 \pm 0.05) \\
\left\langle Z_{\star, \mathrm{B}}\right\rangle_{\mathcal{M}}=(0.42 \pm 0.02) \cdot \log \mathcal{M}_{\star, \mathrm{B}}-(3.26 \pm 0.17) \\
\left\langle Z_{\star, \mathrm{B}}\right\rangle_{\mathcal{M}}=(0.51 \pm 0.02) \cdot \log \mathcal{M}_{\star, \mathrm{T}}-(4.48 \pm 0.23) \\
\left\langle t_{\star, \mathrm{D}}\right\rangle_{\mathcal{M}}=(1.42 \pm 0.12) \cdot \log \mathcal{M}_{\star, \mathrm{T}}-(7.86 \pm 1.25) \\
\left\langle Z_{\star, \mathrm{D}}\right\rangle_{\mathcal{M}}=(0.29 \pm 0.02) \cdot \log \mathcal{M}_{\star, \mathrm{T}}-(2.31 \pm 0.20) \\
\log \mathcal{M}_{\star, \mathrm{B}}=(0.86 \pm 0.06) \cdot\left\langle\delta \mu_{9 \mathrm{G}}\right\rangle+(11.26 \pm 0.09) \\
\log \mathcal{M}_{\star, \mathrm{B}}=(1.20 \pm 0.03) \log \mathcal{M}_{\star, \mathrm{T}}-(2.67 \pm 0.27) \\
\mathcal{M}_{\star, \mathrm{B}} / \mathcal{M}_{\star, \mathrm{T}}=(0.13 \pm 0.02) \cdot \log \mathcal{M}_{\star, \mathrm{T}}-(1.09 \pm 0.18) \\
\left\langle t_{\star, \mathrm{B}}\right\rangle_{\mathcal{M}}=(1.07 \pm 0.08) \cdot\left\langle t_{\star, \mathrm{D}}\right\rangle_{\mathcal{M}}+(0.64 \pm 0.55) \\
\left\langle Z_{\star, \mathrm{B}}\right\rangle_{\mathcal{M}}=(1.42 \pm 0.06) \cdot\left\langle Z_{\star, \mathrm{D}}\right\rangle_{\mathcal{M}}-(0.17 \pm 0.04) \\
\delta t_{\mathrm{BD}}=(0.74 \pm 0.14) \cdot \log \mathcal{M}_{\star, \mathrm{T}}-(6.65 \pm 1.45) \\
\delta Z_{\mathrm{BD}}=(0.22 \pm 0.02) \cdot \log \mathcal{M}_{\star, \mathrm{T}}-(2.18 \pm 0.16)\end{array}$ \\
\hline $\begin{array}{c}\left\langle t_{\star, \mathrm{B}}\right\rangle_{\mathcal{M}}=(2.13 \pm 0.04) \cdot\left\langle\delta \mu_{9 \mathrm{G}}\right\rangle+(12.23 \pm 0.23) \\
\left\langle t_{\star, \mathrm{B}}\right\rangle_{\mathcal{M}}=(2.93 \pm 0.15) \cdot \log \mathcal{M}_{\star, \mathrm{B}}-(19.81 \pm 1.06) \\
\log \Sigma_{\star, \mathrm{B}}=(0.44 \pm 0.03) \cdot \log \mathcal{M}_{\star, \mathrm{B}}+(4.70 \pm 0.30) \\
\log \Sigma_{\star, \mathrm{B}}=(0.16 \pm 0.01) \cdot\left\langle t_{\star, \mathrm{B}}\right\rangle_{\mathcal{M}}+(7.62 \pm 0.10) \\
\log \Sigma_{\star, \mathrm{B}}=(0.34 \pm 0.02) \cdot\left\langle\delta \mu_{9 \mathrm{G}}\right\rangle+(9.58 \pm 0.07) \\
\left\langle Z_{\star, \mathrm{B}}\right\rangle_{\mathcal{M}}=(0.24 \pm 0.01) \cdot \log \mathcal{M}_{\star, \mathrm{B}}-(1.64 \pm 0.11) \\
\left\langle Z_{\star, \mathrm{B}}\right\rangle_{\mathcal{M}}=(0.28 \pm 0.02) \cdot \log \mathcal{M}_{\star, \mathrm{T}}-(2.37 \pm 0.15) \\
\left\langle t_{\star, \mathrm{D}}\right\rangle_{\mathcal{M}}=(2.80 \pm 0.19) \cdot \log \mathcal{M}_{\star, \mathrm{T}}-(21.56 \pm 1.48) \\
\left\langle Z_{\star, \mathrm{D}}\right\rangle_{\mathcal{M}}=(0.21 \pm 0.02) \cdot \log \mathcal{M}_{\star, \mathrm{T}}-(1.61 \pm 0.16) \\
\log \mathcal{M}_{\star, \mathrm{B}}=(0.76 \pm 0.04) \cdot\left\langle\delta \mu_{9 \mathrm{G}}\right\rangle+(10.98 \pm 0.30) \\
\log \mathcal{M}_{\star, \mathrm{B}}=(1.26 \pm 0.02) \log \mathcal{M}_{\star, \mathrm{T}}-(3.27 \pm 0.23) \\
\mathcal{M}_{\star, \mathrm{B}} / \mathcal{M}_{\star, \mathrm{T}}=(0.14 \pm 0.02) \cdot \log \mathcal{M}_{\star, \mathrm{T}}-(1.17 \pm 0.17) \\
\left\langle t_{\star, \mathrm{B}}\right\rangle_{\mathcal{M}}=(1.46 \pm 0.07) \cdot\left\langle t_{\star, \mathrm{D}}\right\rangle_{\mathcal{M}}-(2.23 \pm 0.54) \\
\left\langle Z_{\star, \mathrm{B}}\right\rangle_{\mathcal{M}}=(1.26 \pm 0.06) \cdot\left\langle Z_{\star, \mathrm{D}}\right\rangle_{\mathcal{M}}-(0.04 \pm 0.03) \\
\delta t_{\mathrm{BD}}=(2.35 \pm 0.31) \cdot \log \mathcal{M}_{\star, \mathrm{T}}-(23.42 \pm 2.68) \\
\delta Z_{\mathrm{BD}}=(0.08 \pm 0.01) \cdot \log \mathcal{M}_{\star, \mathrm{T}}-(0.76 \pm 0.12)\end{array}$ & $\begin{array}{c}\left\langle t_{\star, \mathrm{B}}\right\rangle_{\mathcal{M}}=(2.49 \pm 0.07) \cdot\left\langle\delta \mu_{9 \mathrm{G}}\right\rangle+(11.86 \pm 0.37) \\
\left\langle t_{\star, \mathrm{B}}\right\rangle_{\mathcal{M}}=(2.66 \pm 0.20) \cdot \log \mathcal{M}_{\star, \mathrm{B}}-(18.39 \pm 1.45) \\
\log \Sigma_{\star, \mathrm{B}}=(0.42 \pm 0.03) \cdot \log \mathcal{M}_{\star, \mathrm{B}}+(4.93 \pm 0.31) \\
\log \Sigma_{\star, \mathrm{B}}=(0.17 \pm 0.01) \cdot\left\langle t_{\star, \mathrm{B}}\right\rangle_{\mathcal{M}}+(7.74 \pm 0.12) \\
\log \Sigma_{\star, \mathrm{B}}=(0.43 \pm 0.03) \cdot\left\langle\delta \mu_{9 \mathrm{G}}\right\rangle+(9.75 \pm 0.14) \\
\left\langle Z_{\star, \mathrm{B}}\right\rangle_{\mathcal{M}}=(0.43 \pm 0.02) \cdot \log \mathcal{M}_{\star, \mathrm{B}}-(3.40 \pm 0.15) \\
\left\langle Z_{\star, \mathrm{B}}\right\rangle_{\mathcal{M}}=(0.54 \pm 0.02) \cdot \log \mathcal{M}_{\star, \mathrm{T}}-(4.76 \pm 0.20) \\
\left\langle t_{\star, \mathrm{D}}\right\rangle_{\mathcal{M}}=(2.44 \pm 0.21) \cdot \log \mathcal{M}_{\star, \mathrm{T}}-(18.64 \pm 1.65) \\
\left\langle Z_{\star, \mathrm{D}}\right\rangle_{\mathcal{M}}=(0.31 \pm 0.02) \cdot \log \mathcal{M}_{\star, \mathrm{T}}-(2.45 \pm 0.20) \\
\log \mathcal{M}_{\star, \mathrm{B}}=(1.11 \pm 0.08) \cdot\left\langle\delta \mu_{9 \mathrm{G}}\right\rangle+(11.63 \pm 0.57) \\
\log \mathcal{M}_{\star, \mathrm{B}}=(1.25 \pm 0.03) \log \mathcal{M}_{\star, \mathrm{T}}-(3.12 \pm 0.24) \\
\mathcal{M}_{\star, \mathrm{B}} / \mathcal{M}_{\star, \mathrm{T}}=(0.14 \pm 0.02) \cdot \log \mathcal{M}_{\star, \mathrm{T}}-(1.15 \pm 0.19) \\
\left\langle t_{\star, \mathrm{B}}\right\rangle_{\mathcal{M}}=(1.52 \pm 0.12) \cdot\left\langle t_{\star, \mathrm{D}}\right\rangle_{\mathcal{M}}-(2.56 \pm 0.70) \\
\left\langle Z_{\star, \mathrm{B}}\right\rangle_{\mathcal{M}}=(1.63 \pm 0.07) \cdot\left\langle Z_{\star, \mathrm{D}}\right\rangle_{\mathcal{M}}-(0.33 \pm 0.04) \\
\delta t_{\mathrm{BD}}=(3.11 \pm 0.64) \cdot \log \mathcal{M}_{\star, \mathrm{T}}-(31.57 \pm 6.00) \\
\delta Z_{\mathrm{BD}}=(0.23 \pm 0.01) \cdot \log \mathcal{M}_{\star, \mathrm{T}}-(2.25 \pm 0.16)\end{array}$ \\
\hline
\end{tabular}

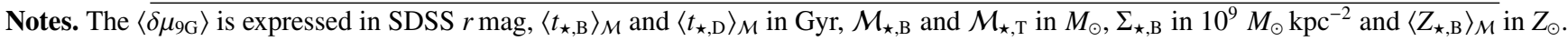

\section{Appendix C: Photometric, evolutionary and physical quantities for the galaxy sample}

The photometric, evolutionary, and physical quantities for the galaxy sample under study (Table C.1) is only available at the CDS.

- Col. 1: name of the galaxy,

- Col. 2: assumed distance in Mpc,

- Col. 3: isophotal radius of the bulge $\left(R_{\mathrm{B}}\right)$ in $\mathrm{kpc}$,

- Col. 4: logarithm of the total stellar mass $\left(\mathcal{M}_{\star, T}\right.$ in $\left.M_{\odot}\right)$,

- Col. 5: logarithm of the present-day stellar mass $\left(\mathcal{M}_{\star, \mathrm{B}}\right.$ in $M_{\odot}$ ) within $R_{\mathrm{B}}$,

- Cols. 6 and 7: mass-weighted stellar age of the bulge $\left(\left\langle t_{\star, \mathrm{B}}\right\rangle_{\mathcal{M}}\right.$ in Gyr) and its dispersion $\left(\sigma\left\langle t_{\star, \mathrm{B}}\right\rangle_{\mathcal{M}}\right)$,
- Cols. 8 and 9: mass-weighted stellar age of the disk $\left(\left\langle t_{\star, \mathrm{D}}\right\rangle_{\mathcal{M}}\right.$ in Gyr) and its dispersion $\left(\sigma\left\langle t_{\star, \mathrm{D}}\right\rangle_{\mathcal{M}}\right)$,

- Cols. 10 and 11: mass-weighted stellar metallicity of the bulge $\left(\left\langle Z_{\star, \mathrm{B}}\right\rangle_{\mathcal{M}}\right.$ in $\left.Z_{\odot}\right)$ and its dispersion $\left(\sigma\left\langle Z_{\star, \mathrm{B}}\right\rangle_{\mathcal{M}}\right)$,

- Cols. 12 and 13: mass-weighted stellar metallicity of the disk $\left(\left\langle Z_{\star, \mathrm{D}}\right\rangle_{\mathcal{M}}\right.$ in $\left.Z_{\odot}\right)$ and its dispersion $\left(\sigma\left\langle Z_{\star, \mathrm{B}}\right\rangle_{\mathcal{M}}\right)$,

- Col. 14: logarithm of the mean stellar mass surface density in the bulge $\left(\Sigma_{\star, \mathrm{B}}\right.$ in $\left.M_{\odot} \mathrm{kpc}^{-2}\right)$ and

- Col. 15: mean $\left\langle\delta \mu_{9 \mathrm{G}}\right\rangle$ ( $r$ mag) within $R_{\mathrm{B}}$. Quantities listed in Cols. 4-15 were obtained from spectral modeling with STARLIGHT of CALIFA V500 data with the Z4 SSP library and refer to the present-day $\mathcal{M}_{\star}$ (cf. Sect. 2.2.2). 